\title{
Synthesen endständig substituierter, konjugierter Polyene ${ }^{1}$
}

Franz Effenberger, ${ }^{*}$ Hubert Schlosser ${ }^{2}$

Institut für Organische Chemie der Universität Stuttgart, Pfaffenwaldring 55, D-7000 Stuttgart 80, Germany

Prof. Dr. Hans-Jürgen Bestmann zum 65. Geburtstag herzlich gewidmet

\section{Syntheses of Terminally Substituted Conjugated Polyenes ${ }^{1}$}

Polyenes and polyenals of the carotenoid type with five, nine and thirteen conjugated double bonds and a variety of terminally substituents like benzenes, naphthalenes, anthracenes, and tetraphenyl porphyrines are synthesized via Wittig reactions starting from crocetin dialdehyde or 2,7-dimethyloctatriendial, respectively. The characterization, and in most cases also the separation, of the $E / Z$-isomers of the polyenes is possible.

In einer Kurzmitteilung ${ }^{1}$ haben wir über die Herstellung konjugierter Polyene mit variierender Kettenlänge und unterschiedlichen Endgruppen berichtet. Von besonderem Interesse sind Donor-Acceptor-substituierte Polyene, da sie für einen intramolekularen Energietransport geeignet sein könnten ${ }^{1}$.

$\mathrm{Zu}$ Beginn unserer Untersuchungen waren Polyene dieses Typs nicht bekannt; inzwischen ist die Darstellung einiger Donor-Acceptor-substituierter, konjugierter Polyene beschrieben worden ${ }^{3,4}$. Wir berichten hier über die präparativen Aspekte der Herstellung dieser Verbindungen, wobei der Struktur-Zuordnung und der Trennung der - durch die Synthese bedingt - anfallenden $E / Z$-Isomeren besondere Bedeutung beigemessen wurde.

In allen Fällen haben wir für die Synthese Segmente natürlich vorkommender Carotinoide ${ }^{5}$ eingesetzt und diese mittels geeigneter Olefinierungs-Methoden ${ }^{6}$ mit den angestrebten Endgruppen verknüpft. Als Polyen-Ausgangsverbindungen wurden Crocetindialdehyd (1), 2,7Dimethyloctatriendial (10) und das aus 10 herstellbare (7-Formyl-2-methyl-2,4,6-octatrienyl)triphenylphosphonium-chlorid (6) verwendet. Die Methyl-Substituenten dieser Verbindungen erhöhen im Vergleich zu den entsprechenden unsubstituierten Polyenen die Stabilität der erwünschten Produkte gegenüber Sauerstoff, Wärme und Belichtung ${ }^{7}$. In der Literatur sind viele Beispiele für den Einsatz der Verbindungen 1, 10 und 6 zum Aufbau von Carotinoiden und Carotinoid-ähnlichen Verbindungen beschrieben ${ }^{5,8}$. Neben wenigen Basen-katalysierten Kondensationsreaktionen werden dort praktisch ausschließlich die Wittig- und die Wittig-Horner-Olefinierung zur Darstellung der Carotinoide benutzt.

\section{Tetramethylheptadecaoctaenale $3 a-h$ und Tetramethyloctadeca- nonaene $4 a-f, 5$}

Läßt man die aus den Phosphonium-Salzen 2a-f mit Butyl-Lithium in etherischer Lösung erhaltenen Ylide zu dem in Dichlormethan vorliegenden Crocetindialdehyd (1) zutropfen, so entstehen in einer Wittig-Reaktion Gemische, die aus den Octaenalen 3a-f (Hauptprodukte) und Nonaenen 4a-f (Nebenprodukte) bestehen und die sich säulenchromatographisch trennen lassen (Schema A, Tabelle 1).<smiles>CC(C=O)=CC=CC(C)=CC=CC=C(C)C=CC=C(C)C=O</smiles>

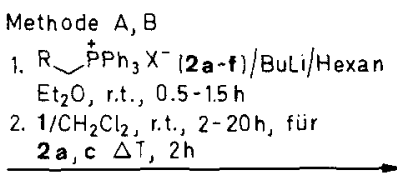<smiles>[R]C=C/C(C)=C/C=C/C(C)=C/C=C/C=C(C)/C=C/C=C(\C)C=O</smiles><smiles>[R]C=CC(C)=CC=CC(C)=CC=CC=C(C)C=CC=C(C)C=[R]</smiles>

4 a-f $4-8 \%$<smiles>CC(/C=C/C=C/C(C)=C/C=C/c1c2ccccc2cc2ccccc12)=C\C=C\C=C(C)\C=C\C=C\c1cccc2ccccc12</smiles>

5

$1+2 \mathrm{~g}, \mathrm{~h}$
Methode $A, B$

1. BuLi/Hexan/Et ${ }_{2} \mathrm{O}$, r.t., in

Methode $C$

$2 \mathrm{e} / \mathrm{BuLi} / \mathrm{Hexan} / \mathrm{Et}_{2} \mathrm{O}$, r.t., in

$2 e+3 d$

2. $1 / \mathrm{CH}_{2} \mathrm{Cl}_{2}$,r.t., $17 \mathrm{~h}$ für $\mathbf{3 g}, 2 \mathrm{~h}$ für $\mathbf{3 h}$

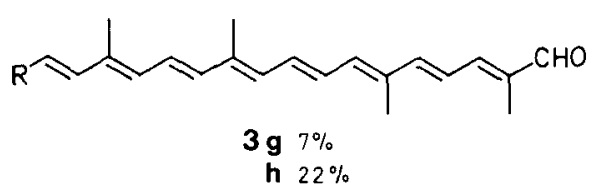

3 g $7 \%$

\begin{tabular}{|c|c|c|c|c|c|c|c|c|}
\hline $1-4$ & $\mathrm{R}$ & $\mathrm{X}$ & $1-4$ & $\mathbf{R}$ & $\mathrm{X}$ & $1-4$ & $\mathrm{R}$ & $\mathrm{X}$ \\
\hline $\mathbf{a}$ & $4-\mathrm{NO}_{2} \mathrm{C}_{6} \mathrm{H}_{4}$ & $\mathrm{Br}$ & $\mathbf{e}$ & 1-Naphthyl & $\mathrm{Cl}$ & $\mathbf{h}$ & 9-(1,4-Dimethyl)anthryl & $\mathrm{Br}$ \\
\hline b & $4-\left(\mathrm{Me}_{2} \mathrm{~N}\right) \mathrm{C}_{6} \mathrm{H}_{4}$ & I & f & $\mathrm{Me}$ & $\mathrm{Br}$ & $\mathbf{i}$ & $2-(5,10,15,20$-Tetraphenyl)- & - \\
\hline c & Ferrocenyl & $\mathrm{I}$ & $\mathbf{g}$ & 2-Anthryl & $\mathrm{Br}$ & & porphyrinyl (TPP) & \\
\hline d & 9-Anthryl & $\mathrm{Br}$ & & & & & & \\
\hline
\end{tabular}


Tabelle 1. Hergestellte Octaenale 3 und Nonaene 4, 5

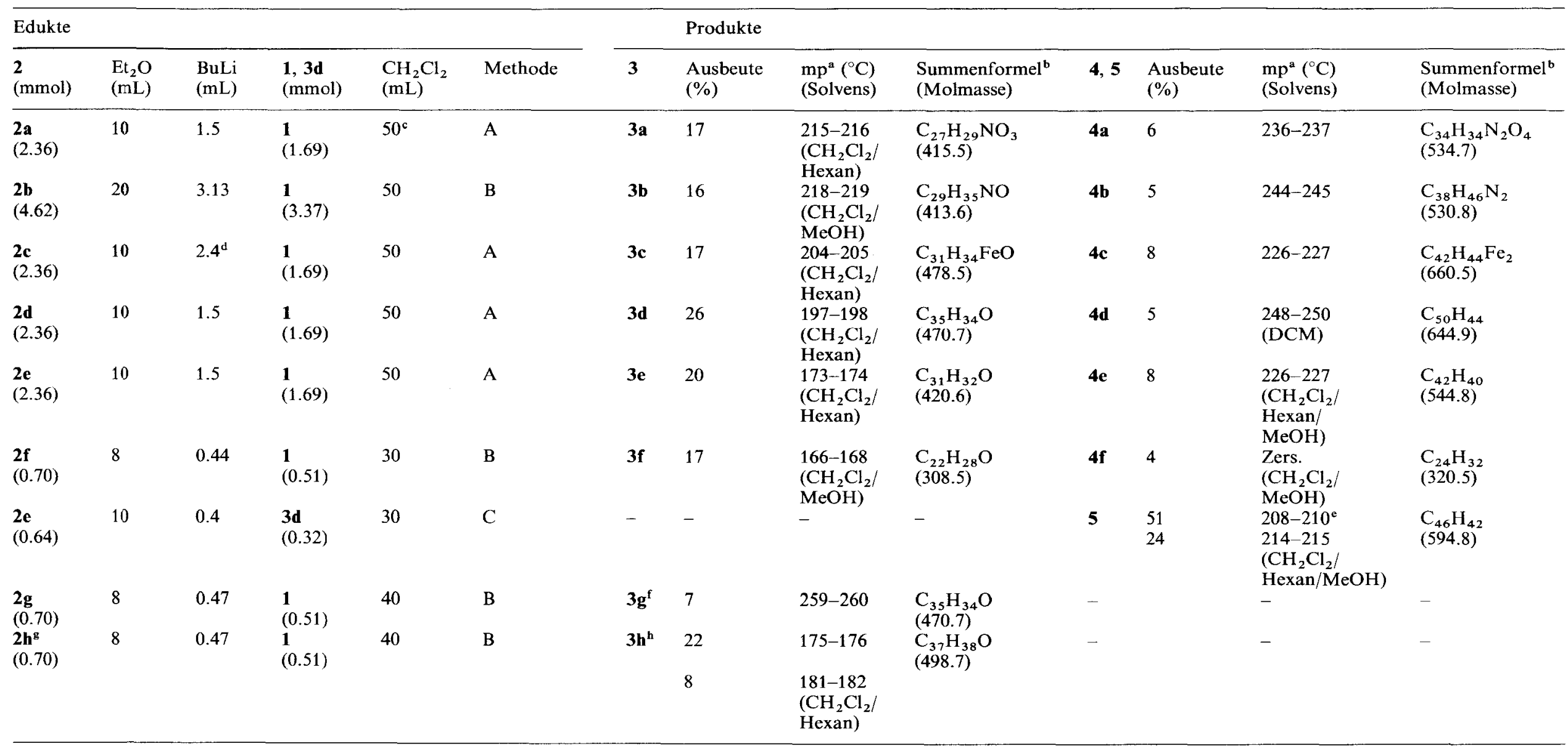

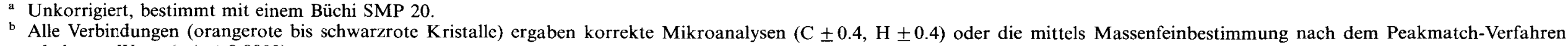
erhaltenen Werte $(m / z \pm 0.0003)$.

c 1,2-Dichlorethan als Lösungsmittel.

d $1 \mathrm{M} \mathrm{BuLi}$ in Hexan.

d $1 \mathrm{M} \mathrm{BuLi}$ in Hexan.
e Aus $\mathrm{CH}_{2} \mathrm{Cl}_{2}$-Lösung durch Zutropfen von $\mathrm{MeOH}$ umgefällt.

f Die Hauptfraktion wurde aus $\mathrm{CH}_{2} \mathrm{Cl}_{2} /$ Hexan umkristallisiert, erneut chromatographiert und umkristallisiert.

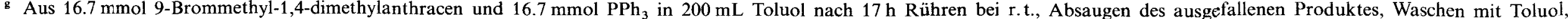
Trocknen i. Hochvak. und Umkristallisieren aus Aceton; Ausbeute: $76 \% 2 \mathrm{~h} ; \mathrm{mp} 231-232{ }^{\circ} \mathrm{C} ; \mathrm{C}_{35} \mathrm{H}_{30} \mathrm{BrP}(561.5)$. ${ }^{1} \mathrm{H}-\mathrm{NMR}\left(\mathrm{CDCl}_{3}\right): \delta=2.71(\mathrm{~s}, 6 \mathrm{H}, 1-, 4-\mathrm{CH})_{3}, 6.68-8.72\left(\mathrm{~m}, 24 \mathrm{H}, \mathrm{CH}_{2}\right.$

$\left.\mathrm{H}_{3 \mathrm{Ph}}, \mathrm{H}_{\text {Anthryl }}\right)$

${ }_{h}$ Die Hauptfraktion wurde erneut über $\mathrm{SiO}_{2}$ mit $\mathrm{CH}_{2} \mathrm{Cl}_{2}$ chromatographiert und aus $\mathrm{CH}_{2} \mathrm{Cl}_{2} / \mathrm{Hexan}$ umkristallisiert. 
Mit den aus den Phosphonium-Salzen $2 \mathbf{g}$, $\mathbf{h}$ entstehenden Yliden reagiert 1 praktisch ausschließlich zu den Octaenalen $3 \mathrm{~g}, \mathrm{~h}$; die entsprechenden Nonaene $\mathbf{4 g}$, $\mathbf{h}$ ließen sich nur dünnschichtchromatographisch in Spuren nachweisen. Das endständig unsymmetrisch substituierte Nonaen 5 erhielten wir durch Umsetzung des Octaenals $\mathbf{3 d}$ mit dem aus 2e entstehenden Ylid (Schema A, Tabelle 1). Die einzige bekannte Verbindung dieses Typs ist das auf vergleichbare Weise hergestellte Di(1naphthyl)-substituierte Nonaen $\mathbf{4} \mathrm{e}^{\mathbf{9}}$.

\section{Isomerenverteilung}

Ausgehend von dem eingesetzten all-E-isomeren Crocetindialdehyd (1) können bei den durchgeführten WittigReaktionen die neu gebildeten Doppelbindungen der Verbindungen 3, 4 und 5 grundsätzlich als $E / Z$ Isomerengemische anfallen. Da aus den PhosphoniumSalzen 2a-g, mit Ausnahme von 2f, Ylide mit moderater Reaktivität entstehen, sollten Gemische mit vergleichbaren $E / Z$-Anteilen anfallen ${ }^{10}$. Da jedoch, ausgehend von 1, für die neu gebildeten Doppelbindungen die benachbarte Methylgruppe eine nicht unbedeutende sterische Hinderung darstellt ${ }^{11,12}$, war $\mathrm{zu}$ erwarten, daß überwiegend all-E-Isomere entstehen, was auch tatsächlich der Fall ist. Die Trennung von E/Z-Isomerengemischen kann, wie Literaturbeispiele zeigen ${ }^{13-15}$, mittels HPLC durchgeführt werden.

Eine Unterscheidung zwischen den all-E- und Z-Isomeren ist anhand der UV/VIS-Spektren möglich ${ }^{16,17}$. Das Hauptabsorptionsmaximum ( $\mathrm{S}_{0} \rightarrow \mathrm{S}_{2}$-Übergang) ist bei den Z-Isomeren zu kürzeren Wellenlängen (ca. $2-5 \mathrm{~nm}$ für eine, ca. $10 \mathrm{~nm}$ für zwei $Z$-Doppelbindungen) verschoben. Bei $Z$-Isomeren kann des weiteren der höherenergetische $\mathrm{S}_{0} \rightarrow \mathrm{S}_{3}$-Übergang erlaubt sein, was zur Ausbildung eines charakteristischen kürzerwelligen sogenannten ,,cis-peaks" führt ${ }^{12,16,17}$.

Die exakte Zuordnung von isomeren Strukturen wurden in einigen Beispielen nach präparativer HPLC-Trennung mittels FT ${ }^{1} \mathrm{H}$-NMR-Spektren (aufgenommen bei $270-400 \mathrm{MHz}$ ) vorgenommen, wodurch insbesondere eine Zuordnung der olefinischen Protonen ermöglicht wird $^{15,17-20}$. In Tabelle 8 sind exemplarisch die Daten der Verbindungen 3a, 4a und 5 angegeben ${ }^{21}$.

Die Bestimmung der Isomerenverteilung in den hergestellten Polyenen 3-5 sowie die Überprüfung der Reinheit der Ausgangsverbindung 1 erfolgte mittels HPLCAnalysen an Kieselgel- bzw. reversed-phase-Säulen, die Zuordnung bzw. Unterscheidung der Isomeren erfolgte durch UV/VIS-Spektren, die nach der stop-flow-Methode von den einzelnen Peaks der Chromatogramme aufgenommen wurden. Die Chromatogramme wurden im UV/VIS-Hauptabsorptionsmaximum des jeweiligen Polyens detektiert und das prozentuale Verhältnis der Isomeren aus den integralen Intensitäten der Peaks ermittelt (Tabelle 2). Bei den nicht über HPLC trennbaren Isomerengemischen und ergänzend zu der angeführten Bestimmung der Isomerenanteile mittels HPLC haben wir aus den Integralen der $300 \mathrm{MHz}{ }^{1} \mathrm{H}$-NMR-Signale für die Endgruppen, die Methyl-Seitengruppen und die olefinischen Protonen die Anteile der all-E-bzw. E/Z-Isomeren in den Produkten abgeschätzt.
Der Ausgangsaldehyd 1 zeigte bei der HPLC-Analyse nur ein Isomer, dessen UV/VIS-, ${ }^{1} \mathrm{H}-\mathrm{NMR}$ - und ${ }^{13} \mathrm{C}$-NMRDaten mit den Literaturwerten für das all-E-Isomere übereinstimmen ${ }^{22}$.

Die Octaenale 3a und $\mathbf{d}-\mathbf{f}$ konnten an Kieselgel getrennt werden, sie zeigten einen all-E-Isomerenanteil zwischen 52 und $84 \%$, nach anschließender Umkristallisation zwischen 89 und $98 \%$ (Tabelle 2). Bei den Verbindungen $\mathbf{3 b}$ und $\mathbf{3 c}$ gelang eine Isomerentrennung mittels HPLC nicht, die Isomerenverteilung wurde daher über die Auswertung der ${ }^{1} \mathrm{H}$-NMR-Spektren vorgenommen. DaB der bei den Octaenalen 3a und 3d-f ${ }^{1} \mathrm{H}-\mathrm{NMR}$-spektroskopisch ermittelte all-E-Isomerenanteil um 2-4\% höher liegt als der durch HPLC ermittelte Wert (Tabelle 2), beruht vermutlich darauf, daß nicht alle bei der Reaktion gebildeten $E / Z$-Isomere im $300 \mathrm{MHz}{ }^{1} \mathrm{H}$-NMR-Spektrum erfaßt werden, so daß letztlich ein höherer all-EIsomerenanteil gefunden wird.

Die neben den Signalen der Aldehyd-, Methyl- und ArylProtonen auftretenden und sich zum Teil stark überlap-

Tabelle 2. Prozentuale Isomerenverteilung der Verbindungen 3, 4 und 5

\begin{tabular}{|c|c|c|}
\hline $\begin{array}{l}\text { Ver- } \\
\text { bindung }\end{array}$ & $\begin{array}{l}\text { Isomerenverteilun } \\
{\left[\text { cis-Peak/ } / \mathrm{S}_{0} \rightarrow \mathrm{S}_{2} \text {, }\right.} \\
\text { all- } E \text {-Isomere }\end{array}$ & $\begin{array}{l}(\%)^{\mathrm{a}} \\
\left.l_{\max }(\mathrm{nm})\right] \\
E / Z \text {-Isomere }\end{array}$ \\
\hline 3a & $\begin{array}{l}75^{b}, 91^{\mathrm{c}}, 95^{\mathrm{c}, \mathrm{d}} \\
{[-/ 474]}\end{array}$ & $\begin{array}{l}9^{\mathrm{b}}, 2^{\mathrm{c}} \quad[-/ 468], \quad 16^{\mathrm{b}}, \quad 7^{\mathrm{c}}, \quad 5^{\mathrm{c}, \mathrm{c}} \\
{[375 / 468]}\end{array}$ \\
\hline 3b & $80^{\mathrm{c} . \mathrm{d}}$ & $20^{\mathrm{c}, \mathrm{d}}$ \\
\hline $3 \mathbf{c}$ & $85^{\mathrm{c}, \mathrm{d}}$ & $15^{\mathrm{c}, \mathrm{d}}$ \\
\hline 3d & $\begin{array}{l}84^{b}, 98^{c}, 100^{c, d} \\
{[-/ 457]}\end{array}$ & $\begin{array}{l}2^{\mathrm{b}}[308 / 451], 6^{\mathrm{b}}[309 / 452], 5^{\mathrm{b}}, 2^{\mathrm{c}} \\
{[326 / 447], 3^{\mathrm{b}}[326 / 449]}\end{array}$ \\
\hline $3 \mathbf{e}$ & $\begin{array}{l}70^{b}, 98^{c}, 100^{c, d} \\
{[-/ 460]}\end{array}$ & $20^{\mathrm{b}}[-/ 448], 10^{\mathrm{b}}, 2^{\mathrm{c}}[350 / 455]$ \\
\hline 3f & $\begin{array}{l}52^{b}, 89^{\mathrm{c}}, 91^{\mathrm{c}, \mathrm{d}} \\
{[-/ 460]}\end{array}$ & $\begin{array}{l}2^{\mathrm{b}}, 4^{\mathrm{c}}, 9^{\mathrm{c}, \mathrm{d}}[-/ 459], 1^{\mathrm{b}}, 1^{\mathrm{c}}[-/ 442] \\
1^{\mathrm{b}}[-/ 446], 36^{\mathrm{b}}, 4^{\mathrm{c}}[-/ 445], 5^{\mathrm{b}}, 1^{\mathrm{c}} \\
{[-/ 446], 2^{\mathrm{b}}, 1^{\mathrm{c}}[347 / 452], 1^{\mathrm{b}}} \\
{[325 / 439]}\end{array}$ \\
\hline 3g & $92^{\mathrm{e}}[-/ 477]$ & $3^{e}[-/ 469], 5^{e}[345 / 470]$ \\
\hline 3h & $\begin{array}{l}86^{\mathrm{e}}, 99^{\mathrm{e}}, 86^{\mathrm{e}, \mathrm{d}} \\
{[-/ 453]}\end{array}$ & $14^{\mathrm{e}}, 1^{\mathrm{e}}, 14^{\mathrm{e}, \mathrm{d}}[-/ 446]$ \\
\hline $4 \mathbf{a}$ & $\begin{array}{l}74^{\mathrm{c}}, 75^{\mathrm{c}, \mathrm{d}} \\
{[-/ 499]}\end{array}$ & $20^{\mathrm{c}}, 25^{\mathrm{c}, \mathrm{d}}[-/ 493], 6^{\mathrm{c}}[416 / 494]$ \\
\hline $4 b$ & $70^{\mathrm{c}, \mathrm{d}}$ & $30^{\mathrm{c}, \mathrm{d}}$ \\
\hline $4 c$ & $71^{\mathrm{c}, \mathrm{d}}$ & $29^{\mathrm{c}, \mathrm{d}}$ \\
\hline 4d & $87^{\mathrm{c}, \mathrm{d}}$ & $-^{c \cdot d}$ \\
\hline $4 e$ & $\begin{array}{l}60^{\mathrm{c}}, 96^{\mathrm{c}}, 100^{\mathrm{c}, \mathrm{d}} \\
{[-/ 473]}\end{array}$ & $\begin{array}{l}32^{\mathrm{c}}, 4^{\mathrm{c}}[375 / 468], 7^{\mathrm{c}}[375 / 470], 1^{\mathrm{c}} \\
{[362 / 460]}\end{array}$ \\
\hline $4 f$ & $98^{\mathrm{c}, \mathrm{d}}$ & $2^{c, d}$ \\
\hline 5 & $\begin{array}{l}73^{b}, 84^{c}, 85^{c, d} \\
{[-/ 470]}\end{array}$ & $\begin{array}{l}7^{\mathrm{b}}, 5^{\mathrm{c}}[356 / 467], 19^{\mathrm{b}}, 11^{\mathrm{c}}, 15^{\mathrm{c}, \mathrm{d}} \\
{[356 / 468], 1^{\mathrm{b}}[-/ 459]}\end{array}$ \\
\hline
\end{tabular}

a Die Isomerenverteilung wurde mittels einer HPLC-Anlage LDC/Milton Roy Gradienten-System bestehend aus zwei LDC/Milton Roy Constametric III Pumpen, Gradientenmischer, Säulenschaltung, LDC/Milton Roy Steuereinheit MP $3000 \mathrm{E}$ und einem UV/VIS-Detektor (Fa. Uvikon) Typ $720 \mathrm{LC}$, Säule SI-100, $7 \mu \mathrm{m}, 250 \times 4,6 \mathrm{~mm}$, Hexan/EtOAc als Eluierungsmittel $(95: 5 \rightarrow 92: 8)$ und flow $(1.5-2.0 \mathrm{~mL} / \mathrm{min})$ bestimmt.

${ }^{b}$ Nach Chromatographie.

c Anreicherung nach Chromatographie und Umkristallisation bzw. Umfällung.

d Bestimmt mittels ${ }^{1} \mathrm{H}$-NMR-Spektroskopie.

e Anreicherung nach wiederholter Chromatographie, Umkristallisation bzw. Umfällung. 
penden Signale der olefinischen Protonen konnten durch Vergleich mit den ${ }^{1}$ H-NMR-Spektren von 8'-Apocarotinalen ${ }^{19,20}$ unter Berücksichtigung des Einflusses der aromatischen Endgruppen weitgehend exakt zugeordnet werden. ${ }^{3} J_{\mathrm{H}, \mathrm{H}}$-Kopplungskonstanten zwischen 15.52 und $16.14 \mathrm{~Hz}$ bestätigen die $E$-Konfiguration der bei den Wittig-Reaktionen gebildeten Doppelbindung zwischen C-16 und C-17 in den Verbindungen $3 \mathbf{a}-\mathbf{c}$ und $\mathbf{3 e - h}$; bei 3d ist eine Zuordnung dieser Protonensignale wegen der Überlagerung mit anderen olefinischen Protonen und mit den Protonensignalen des 9-Anthryl-Restes nicht möglich. Bei den Nonaenen 4a-f und 5 wurden die $E / Z$ Isomerenanteile in gleicher Weise mittels HPLC bzw. ${ }^{1} \mathrm{H}$ NMR-spektroskopisch ermittelt und Strukturzuordnungen durch Analyse der ${ }^{1} \mathrm{H}$-NMR-Spektren getroffen. Das nach Chromatographie und Umfällen erhaltene Nonaen 5 ließ sich z. B. mittels HPLC in vier Isomere auftrennen: das all-E-Isomer stellt mit $73 \%$ das Hauptprodukt dar, von den drei $E / Z$-Isomeren überwiegt eines mit $19 \%$ (Tabelle 2).

\section{Hexamethylpentacosadodecaenale $7 \mathrm{a}-\mathrm{e}$ und Hexamethylhexaco-} satridecaene 8d, e, 9

Wie bereits erwähnt, sollte für die Untersuchungen eines intramolekularen Energietransfers in konjugierten Polyenen auch die Kettenlänge der Polyene variiert werden. Konjugierte Polyene mit zwölf bzw. dreizehn Doppelbindungen, die endständig mit Donor- bzw. AcceptorMolekülen verknüpft sind, haben wir ausgehend von den unter 1. beschriebenen Polyenen 3a-e über WittigReaktionen mit dem aus dem Phosphonium-Salz 6 erhaltenen Ylid hergestellt.

Das Phosphonium-Salz 6 wurde aus 2,7-Dimethyl-octatriendial (10) hergesellt ${ }^{23}$. Zur Vermeidung von Nebenreaktionen wurde die Aldehydgruppe in 6 zunächst acetalisiert, das gebildete Acetal in situ mit Natriummethanolat/Methanol in das Ylid übergeführt und dieses dann jeweils mit den in Dichlormethan gelösten Aldehyden

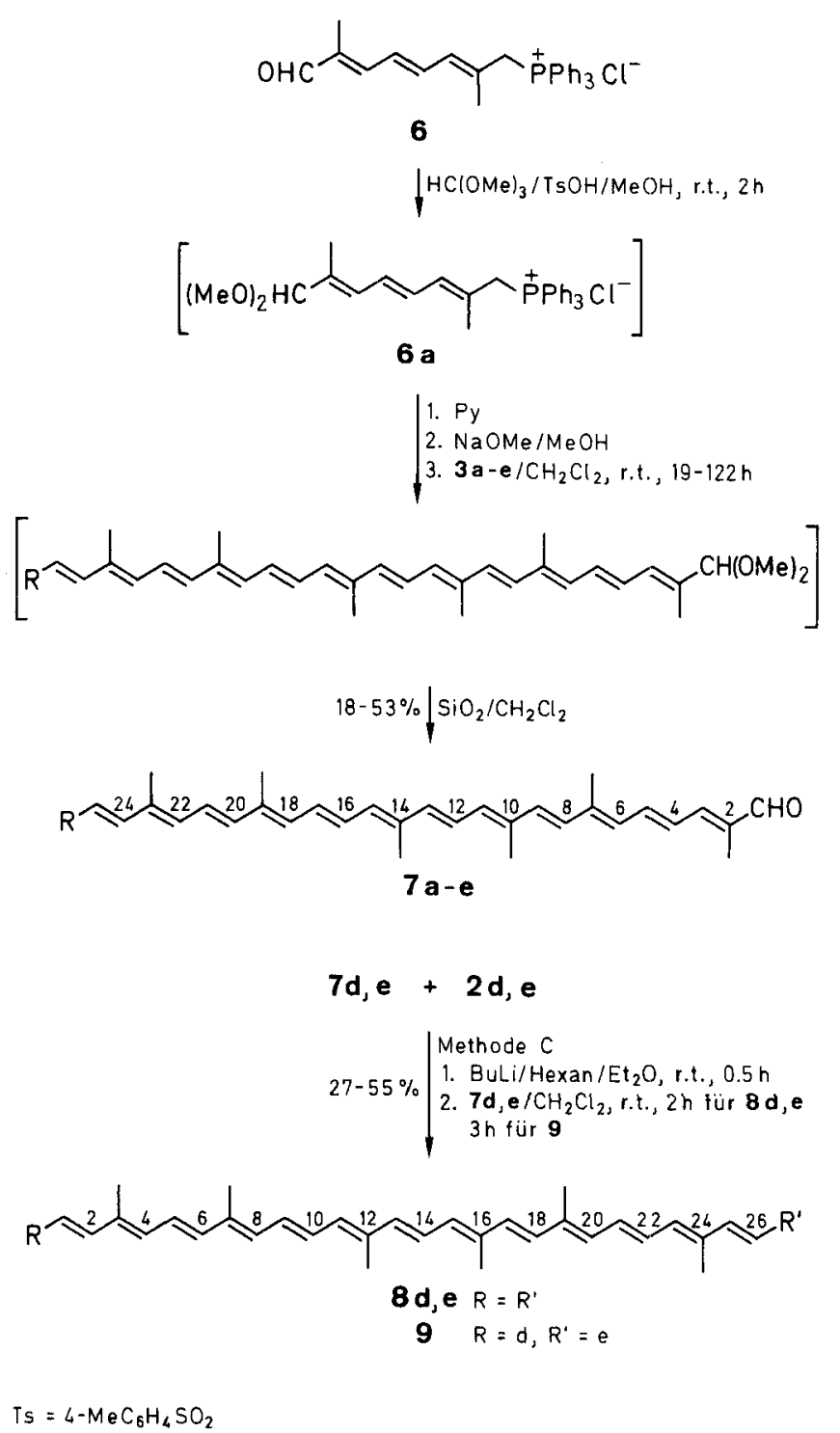

Schema B

Tabelle 3. Hergestellte Pentacosadodecaenale 7a-e

\begin{tabular}{|c|c|c|c|c|c|c|c|c|}
\hline \multicolumn{4}{|l|}{ Edukte } & \multirow{2}{*}{$\begin{array}{l}\text { Pro- } \\
\text { dukt }\end{array}$} & \multirow{2}{*}{$\begin{array}{l}\text { Aus- } \\
\text { beute } \\
(\%)\end{array}$} & \multirow{2}{*}{$\begin{array}{l}\operatorname{mp}^{\mathrm{b}}\left({ }^{\circ} \mathrm{C}\right) \\
\text { (Solvens) }\end{array}$} & \multirow{2}{*}{$\begin{array}{l}\text { all- } E^{\mathrm{c}} \\
(\%)\end{array}$} & \multirow{2}{*}{$\begin{array}{l}\text { Summenformel } \\
\text { (Molmasse) }\end{array}$} \\
\hline $\begin{array}{l}6^{\mathrm{a}} \\
(\mathrm{mmol})\end{array}$ & $\begin{array}{l}1 \mathrm{~N}-\mathrm{NaOMe} \\
(\mathrm{mL})\end{array}$ & $\begin{array}{l}3 \\
(\mathrm{mmol})\end{array}$ & $\begin{array}{l}\mathrm{CH}_{2} \mathrm{Cl}_{2} \\
(\mathrm{~mL})\end{array}$ & & & & & \\
\hline $\begin{array}{l}6 \mathrm{a} \\
(0.50)\end{array}$ & 0.6 & $\begin{array}{l}\mathbf{3 a} \\
(0.24)\end{array}$ & 25 & $7 \mathbf{a}$ & 18 & $\begin{array}{l}222-223 \\
\text { (Benzol) }\end{array}$ & 64 & $\begin{array}{l}\mathrm{C}_{37} \mathrm{H}_{41} \mathrm{NO}_{3} \\
(547.7)\end{array}$ \\
\hline $\begin{array}{l}\mathbf{6 b} \\
(0.50)\end{array}$ & 0.5 & $\begin{array}{l}\text { 3b } \\
(0.24)\end{array}$ & 25 & $7 \mathbf{b}$ & 47 & $\begin{array}{l}238-239 \\
\left(\mathrm{CH}_{2} \mathrm{Cl}_{2} / \text { Hexan }\right)\end{array}$ & 62 & $\begin{array}{l}\mathrm{C}_{39} \mathrm{H}_{47} \mathrm{NO} \\
(545.8)\end{array}$ \\
\hline $\begin{array}{l}6 c \\
(0.40)\end{array}$ & 0.5 & $\begin{array}{l}3 c \\
(0.19)\end{array}$ & 25 & $7 c$ & 47 & $\begin{array}{l}229-230 \\
\text { (Dichlorethan/ } \\
\mathrm{Et}_{2} \mathrm{O} \text { ) }\end{array}$ & 62 & $\begin{array}{l}\mathrm{C}_{41} \mathrm{H}_{46} \mathrm{FeO} \\
(610.7)\end{array}$ \\
\hline $\begin{array}{l}\text { 6d } \\
(0.50)\end{array}$ & 0.5 & $\begin{array}{l}\mathbf{3 d} \\
(0.21)\end{array}$ & 30 & $7 d$ & 50 & $\begin{array}{l}208-209 \\
\left(\mathrm{CH}_{2} \mathrm{Cl}_{2} / \text { Hexan }\right)\end{array}$ & 74 & $\begin{array}{l}\mathrm{C}_{45} \mathrm{H}_{46} \mathrm{O} \\
(602.9)\end{array}$ \\
\hline $\begin{array}{l}\mathbf{6 e} \\
(0.50)\end{array}$ & 0.5 & $\begin{array}{l}3 \mathbf{e} \\
(0.24)\end{array}$ & 25 & $7 e$ & 53 & $\begin{array}{l}201-203 \\
\left(\mathrm{CH}_{2} \mathrm{Cl}_{2} / \text { Hexan }\right)\end{array}$ & 78 & $\begin{array}{l}\mathrm{C}_{41} \mathrm{H}_{44} \mathrm{O} \\
(552.8)\end{array}$ \\
\hline
\end{tabular}

a Jeweils in $5 \mathrm{~mL} \mathrm{MeOH}$ mit $0.91 \mathrm{mmol}$ Orthoameisensäure-trimethylester, $1 \mathrm{mg}$ TsOH und $0.1 \mathrm{~mL}$ Pyridin.

b Unkorrigiert, bestimmt mit einem Büchi SMP 20.

c Isomerenanteil der umkristallisierten Produkte nach ${ }^{1} \mathrm{H}-\mathrm{NMR}$.

d Alle Verbindungen (rotbraune bis schwarze Feststoffe) ergaben korrekte Mikroanalysen $(\mathrm{C} \pm 0.40, \mathrm{H} \pm 0.40)$ oder die nach der Massenfeinbestimmung nach dem Peakmatch-Verfahren erhaltenen Werte $(m / z \pm 0.0003)$. 
3a-e umgesetzt. Nach chromatographischer Aufarbeitung der Reaktionsgemische über Kieselgel als Säulenmaterial wurden die Pentacosadodecaenale 7a-e mit zwölf konjugierten Doppelbindungen in guten Ausbeuten erhalten (Tabelle 3). Daß hierbei primär die Acetale der Aldehyde 7 entstehen, die dann bei der Aufarbeitung über Kieselsäure hydrolysiert werden, zeigte die Verfolgung der Reaktion mittels Dünnschichtchromatographie an neutralem Aluminiumoxid.

Umsetzungen der 9-Anthryl-7 d und 1-Naphthylpolyenale $7 \mathbf{e}$ mit den Phosphoniumsalzen 2d, $\mathbf{e}$ - ebenfalls unter den Bedingungen einer Wittig-Reaktion - führten zu den endständig Anthryl- und Naphthyl-substituierten Tridecaenen 8d, e, 9 mit jeweils 13 konjugierten Doppelbindungen (Schema B, Tabelle 4).

\section{Isomerenverteilung}

Nachdem der Dialdehyd 10 als reine all-E-Verbindung eingesetzt wurde ${ }^{24}$ und für das hieraus hergestellte Phosphonium-Salz 6 aus den Integralverhältnissen der Signale des Aldehydprotons sowie der dem Phosphor benachbarten Methylenprotonen im $300 \mathrm{MHz}{ }^{1} \mathrm{H}-\mathrm{NMR}$-Spektrum ein all-E-Anteil von ca. $70 \%$ ermittelt wurde, müssen bei der Hydriedrung, der Halogenierung oder der Phosphonium-Salz-Bildung zu 6 Isomerisierungsreaktionen eingetreten sein. Die eingesetzten Aldehyde 3a-e wurden mit den in Tabelle 2 aufgeführten all-E-Isomerenanteilen (80-98\%) eingesetzt. Für die aus diesen Verbindungen ( 3 und 6) hergestellten Polyenale $7 \mathbf{a}-\mathbf{e}$ waren daher ebenfalls Isomerengemische zu erwarten, für die Tridecaene 8d, e, 9 - bedingt durch die große Raumerfüllung der Endgruppe - eine bevorzugte Bildung der all-E-Isomeren.

Alle Versuche, die erhaltenen Isomerengemische mittels HPLC zu trennen, schlugen fehl, so daß die Strukturzuordnung mit Hilfe der $300 \mathrm{MHz}{ }^{1} \mathrm{H}-\mathrm{NMR}$-Spektren (siehe Beispiele in Tabelle 8) erfolgen mußte.

Bei den Polyenalen 7a-e stimmt die Hauptabsorption der Aldehydprotonen zwischen $\delta=9.455$ und $9.458 \mathrm{sehr}$ gut mit den bei gleichen Werten erscheinenden Signalen der Aldehydprotonen der all-E-Octaenale 3 überein, so daß diese Signale den all-E-isomeren Dodecaenalen 7 zugeordnet wurden (s. Tabellen 3,8); die zwischen $\delta=9.460$ und 9.464 erscheinenden Aldehydprotonensignale geringerer Intensität der Verbindungen 7, die auch bei $Z$-Carotinalen auftreten, ${ }^{19}$ wurden dann den $E / Z$ isomeren Dodecaenalen 7 zugeordnet. Auch die Auswertung der Signale der olefinischen Protonen und der Protonen der Endgruppen ergaben eine Isomerenverteilung für die Verbindungen $\mathbf{7 a - e}$, die mit denen, die über die Auswertung der Aldehydprotonensignale erhalten wurden, übereinstimmen.

Die $E$-Konfiguration der neu gebildeten Doppelbindungen in den Verbindungen 8e und 9 wird durch die ${ }^{3} J_{\mathrm{H}, \mathrm{H}^{-}}$ Kopplung bei $15.79 \mathrm{bzw} .16 .02 \mathrm{~Hz}$ bestätigt (Tabelle 8).

\section{2,7-Dimethylnonatetraenale 11d, e, g, h und 3,8-Dimethyldeca- pentaene 12d, e, 13}

Nach der Synthese endständig substituierter Polyene mit dreizehn bzw. neun konjugierten Doppelbindungen haben wir ausgehend von dem Octatriendialdehyd 10 auch die Aryl-substituierten Tetraenale $\mathbf{1 1 d} \mathbf{d}, \mathbf{e}, \mathbf{g}, \mathbf{h}$ sowie die Diaryl-substituierten Pentaene 12d, e, 13 hergestellt (Schema C, Tabelle 5).

Die invers geführte Wittig-Reaktion führte wie bei den Reaktionen mit Crocetindialdehyd (1) zu Gemischen aus 11 und 12, wobei letztere nur als Nebenprodukte in kleinen Mengen erhalten wurden, die sich chromatographisch leicht abtrennen ließen. Bei den Umsetzungen der aus den Phosphonium-Salzen 2 $\mathbf{g}$, $\mathbf{h}$ entstehenden Ylide mit 10 wurden die Aryl-substituierten Tetraenale $11 \mathrm{~g}, \mathbf{h}$ erhalten, während bei der Wittig-Reaktion des aus $2 \mathrm{e}$ entstehenden Ylids mit dem Tetraenal 11 d das endständig Anthryl- und Naphthyl-substituierte Pentaen 13 isoliert werden konnte (Ausbeute 73\%).

\section{Isomerenverteilung}

$\mathrm{Da}$ der verwendete Dialdehyd $\mathbf{1 0}$ als reines all-E-Isomer zur Verfügung stand und bei den in diesem Kapitel beschriebenen Olefinierungsreaktionen nur die sterisch

Tabelle 4. Hergestellte Hexacosatridecaene 8d, e, 9

\begin{tabular}{|c|c|c|c|c|c|c|c|c|c|}
\hline \multicolumn{5}{|l|}{ Edukte } & \multirow{2}{*}{$\begin{array}{l}\text { Pro- } \\
\text { dukt }\end{array}$} & \multirow{2}{*}{$\begin{array}{l}\text { Aus- } \\
\text { beute } \\
(\%)\end{array}$} & \multirow{2}{*}{$\begin{array}{l}m p^{a}\left({ }^{\circ} \mathrm{C}\right) \\
\text { (Solvens) }\end{array}$} & \multirow{2}{*}{$\begin{array}{l}\text { all-E } E^{\mathrm{b}} \\
(\%)\end{array}$} & \multirow{2}{*}{$\begin{array}{l}\text { Summen- } \\
\text { formel }^{\mathfrak{c}} \\
\text { (Molmasse) }\end{array}$} \\
\hline $\begin{array}{l}2 \\
\text { (mmol) }\end{array}$ & $\begin{array}{l}\mathrm{Et}_{2} \mathrm{O} \\
(\mathrm{mL})\end{array}$ & $\begin{array}{l}\mathrm{BuLi} \\
(\mathrm{mL})\end{array}$ & $\begin{array}{l}7 \\
(\mathrm{mmol})\end{array}$ & $\begin{array}{l}\mathrm{CH}_{2} \mathrm{Cl}_{2} \\
(\mathrm{~mL})\end{array}$ & & & & & \\
\hline $\begin{array}{l}2 \mathbf{d} \\
(0.20)\end{array}$ & 10 & 0.13 & $\begin{array}{l}7 \mathbf{d} \\
(0.10)\end{array}$ & 30 & $8 d$ & 48 & 191-193 $\left(\mathrm{CH}_{2} \mathrm{Cl}_{2} / \mathrm{MeOH}\right)^{\mathrm{d}}$ & & $\begin{array}{l}\mathrm{C}_{60} \mathrm{H}_{56} \\
(777.1)\end{array}$ \\
\hline $\begin{array}{l}2 \mathrm{e} \\
(0.18)\end{array}$ & 5 & 0.11 & $\begin{array}{l}7 \mathbf{e} \\
(0.09)\end{array}$ & 30 & $8 e$ & $\begin{array}{l}27 \\
84\end{array}$ & $\begin{array}{l}197-198\left(\mathrm{CH}_{2} \mathrm{Cl}_{2} / \mathrm{Hexan}\right) \\
195-196\left(\mathrm{CH}_{2} \mathrm{Cl}_{2} / \mathrm{MeOH}\right)^{\mathrm{d}}\end{array}$ & 78 & $\begin{array}{l}\mathrm{C}_{52} \mathrm{H}_{52} \\
(676.9)\end{array}$ \\
\hline $\begin{array}{l}2 \mathrm{e} \\
(0.16)\end{array}$ & 5 & 0.10 & $\begin{array}{l}7 \mathbf{d} \\
(0.80)\end{array}$ & 20 & 9 & $\begin{array}{l}55 \\
48 \\
30\end{array}$ & $\begin{array}{l}203-204\left(\mathrm{CH}_{2} \mathrm{Cl}_{2} / \mathrm{Hexan}\right) \\
200-202\left(\mathrm{CH}_{2} \mathrm{Cl}_{2} / \mathrm{MeOH}\right)^{\mathrm{d}} \\
207-208\left(\mathrm{CH}_{2} \mathrm{Cl}_{2} / \text { Hexan }\right)\end{array}$ & $\begin{array}{l}80 \\
80\end{array}$ & $\begin{array}{l}\mathrm{C}_{56} \mathrm{H}_{54} \\
(727.0)\end{array}$ \\
\hline
\end{tabular}

\footnotetext{
ankorrigiert, bestimmt mit einem Büchi SMP 20.

b Isomerenanteil der umkristallisierten Produkte nach ${ }^{1} \mathrm{H}-\mathrm{NMR}$.

Alle Verbindungen (rotbraune bis schwarzbraune Feststoffe) ergaben korrekte Mikroanalyse (für 9: $\mathrm{C} \pm 0.40, H \pm 0.40$ ) oder die mittels

Massenfeinbestimmung nach dem Peakmatch-Verfahren erhaltenen Werte $(\mathrm{m} / z \pm 0.0012)$.
Nach Lösen der ersten Hauptfraktion in $\mathrm{CH}_{2} \mathrm{Cl}_{2}$, Ausfällen mit $\mathrm{MeOH}$, Abfiltrieren, Waschen mit MeOH und Trocknen i. Hochvak.
} 
anspruchsvollen Naphthylmethyl- und Anthrylmethylphosphonium-Salze 2d, e, g, h eingesetzt wurden, war bei den Produkten ein hoher Anteil an all-E-Isomeren zu erwarten. Mit Ausnahme von 11g wurden alle hergestellten Verbindungen 11, 12 und 13 nach chromatographischer Reinigung und Umkristallisation mit einem Anteil von $97-100 \%$ als all-E-Isomere erhalten (Tabelle 7). Bei dem sterisch weniger anspruchsvollen 2-Anthryltetraenal $11 \mathrm{~g}$ fielen nach zweimaliger Chromatographie und Umkristallisation $78 \%$ all-E- und zwei $E / Z$-Isomere mit $13 \%$ und $9 \%$ an, nach dreimaliger Chromatographie und zweimaliger Umkristallisation konnte das all$E$-Isomer $11 \mathrm{~g}$ auf $97 \%$ angereichert werden (Tabelle 7). Die ${ }^{1} \mathrm{H}$-NMR-spektroskopisch bestimmte Isomerenverteilung bestätigt die durch HPLC erhaltenen Werte. Bei den umkristallisierten Produkten traten nur die Signale eines Isomers auf, wobei die ${ }^{3} J_{\mathrm{H}, \mathrm{H}^{-}}$-Kopplungskonstanten der nicht methylsubstituierten Doppelbindungen mit 14.34 bis $16.20 \mathrm{~Hz}$ die E-Konfiguration belegen (Tabelle $8)$.

Tabelle 5. Hergestellte Nonatetraenale 11d, e, g, h und Decapentaene 12d, e, 13

\begin{tabular}{|c|c|c|c|c|c|c|c|c|c|c|c|c|c|}
\hline \multicolumn{6}{|l|}{ Edukte } & \multicolumn{8}{|c|}{ Produkte } \\
\hline $\begin{array}{l}2 \\
(\mathrm{mmol})\end{array}$ & $\begin{array}{l}\mathrm{Et}_{2} \mathrm{O} \\
(\mathrm{mL})\end{array}$ & $\begin{array}{l}\mathrm{BuLi} \\
(\mathrm{mL})\end{array}$ & $\begin{array}{l}\text { 10/11d } \\
(\mathrm{mmol})\end{array}$ & $\begin{array}{l}\mathrm{CH}_{2} \mathrm{Cl}_{2} \\
(\mathrm{~mL})\end{array}$ & $\begin{array}{l}\text { Me- } \\
\text { thode }\end{array}$ & 11 & $\begin{array}{l}\text { Aus- } \\
\text { beute } \\
(\%)\end{array}$ & $\begin{array}{l}\mathrm{mp}^{\mathrm{a}} \\
\left({ }^{\circ} \mathrm{C}\right) \\
\text { (Solvens) }\end{array}$ & $\begin{array}{l}\text { Summen- } \\
\text { formel }^{\mathrm{b}} \\
\text { (Molmasse) }^{\text {Molmo }}\end{array}$ & $12 / 13$ & $\begin{array}{l}\text { Aus- } \\
\text { beute } \\
(\%)\end{array}$ & $\begin{array}{l}m p^{a} \\
\left({ }^{\circ} \mathrm{C}\right) \\
\text { (Solvens) }\end{array}$ & $\begin{array}{l}\text { Summen- } \\
\text { formel }^{b} \\
\text { (Molmasse) }\end{array}$ \\
\hline $\begin{array}{l}\mathbf{2 d} \\
(1.70)\end{array}$ & 10 & 1.70 & $\begin{array}{l}\mathbf{1 0} \\
(1.20)\end{array}$ & 40 & B & $11 d$ & 53 & $\begin{array}{l}216-218 \\
\text { (Toluol/ } \\
\text { MeOH, } \\
1: 1 \text { ) }\end{array}$ & $\begin{array}{l}\mathrm{C}_{25} \mathrm{H}_{22} \mathrm{O} \\
(338.4)\end{array}$ & $12 d$ & 9 & $\begin{array}{l}\text { 261-263 } \\
\text { (Toluol) }\end{array}$ & $\begin{array}{l}\mathrm{C}_{40} \mathrm{H}_{32} \\
(512.7)\end{array}$ \\
\hline $\begin{array}{l}\mathbf{2 e} \\
(1.28)\end{array}$ & 8 & 1.28 & $\begin{array}{l}\mathbf{1 0} \\
(0.91)\end{array}$ & 30 & B & $11 \mathrm{e}$ & 21 & $\begin{array}{l}104-106 \\
\text { (Hexan) }\end{array}$ & $\begin{array}{l}\mathrm{C}_{21} \mathrm{H}_{20} \mathrm{O} \\
(288.4)\end{array}$ & $12 \mathrm{e}$ & 13 & $\begin{array}{l}211-213 \\
\text { (Toluol/ } \\
\mathrm{MeOH}, \\
1: 1)\end{array}$ & $\begin{array}{l}\mathrm{C}_{32} \mathrm{H}_{28} \\
(412.6)\end{array}$ \\
\hline $\begin{array}{l}2 \mathrm{~g} \\
(1.28)\end{array}$ & 8 & 1.28 & $\begin{array}{l}10 \\
(0.91)\end{array}$ & 30 & B & $11 \mathrm{~g}$ & $\begin{array}{l}52^{\mathrm{c}} \\
21^{\mathrm{d}}\end{array}$ & $\begin{array}{l}210-211 \\
215-216\end{array}$ & $\begin{array}{l}\mathrm{C}_{25} \mathrm{H}_{22} \mathrm{O} \\
(338.4)\end{array}$ & - & - & - & - \\
\hline $\begin{array}{l}2 \mathrm{~h} \\
(1.28)\end{array}$ & 8 & 1.28 & $\begin{array}{l}\mathbf{1 0} \\
(0.91)\end{array}$ & 35 & B & $11 \mathrm{~h}$ & $33^{e}$ & $\begin{array}{l}133-134 \\
\text { (Hexan) }\end{array}$ & $\begin{array}{l}\mathrm{C}_{27} \mathrm{H}_{26} \mathrm{O} \\
(366.5)\end{array}$ & - & - & - & - \\
\hline $\begin{array}{l}\mathbf{2 e} \\
(0.36)\end{array}$ & 8 & 0.36 & $\begin{array}{l}11 \mathrm{~d} \\
(0.18)\end{array}$ & 20 & $\mathrm{C}$ & - & - & - & - & $13^{f}$ & 72 & $\begin{array}{l}208-209 \\
\text { (Toluol/ } \\
\mathrm{MeOH}, \\
1: 1 \text { ) }\end{array}$ & $\begin{array}{l}\mathrm{C}_{36} \mathrm{H}_{30} \\
(462.6)\end{array}$ \\
\hline
\end{tabular}

a Unkorrigiert, bestimmt mit einem Büchi SMP 20.

b Alle Verbindungen (gelbe bis orangerote Kristalle) ergaben korrekte Mikroanalysen: $\mathrm{C} \pm 0.40, \mathrm{H} \pm 0.40$.

c Nach nochmaligem Chromatographieren an $\mathrm{SiO}_{2}$ mit $\mathrm{CH}_{2} \mathrm{Cl}_{2}$ als Laufmittel und Umkristallisieren aus $\mathrm{CH}_{2} \mathrm{Cl}_{2} / \mathrm{MeOH}$

¿ Nach Wiederholung von ${ }^{\mathrm{c}}$.

e Nach Umkristallisation der Hauptfraktion.

f Nach Umkristallisation der ersten Hauptfraktion.

Tabelle 6. Hergestellte TPP-substituierte Pentaene 15d, $\mathbf{g}, \mathbf{h}$ und Nonaene 16d, $\mathbf{g}, \mathbf{h}$

\begin{tabular}{|c|c|c|c|c|c|c|c|}
\hline \multicolumn{5}{|l|}{ Edukte } & \multirow[b]{2}{*}{ Produkt } & \multirow[b]{2}{*}{ Ausbeute $(\%)$} & \multirow[b]{2}{*}{ Summenformel $^{\mathrm{b}}$ (Molmasse) } \\
\hline $\begin{array}{l}2 \\
(\mathrm{mmol})\end{array}$ & $\begin{array}{l}\mathrm{Et}_{2} \mathrm{O} \\
(\mathrm{mL})\end{array}$ & $\begin{array}{l}\text { BuLi } \\
(\mathrm{mL})\end{array}$ & $\begin{array}{l}\mathbf{1 1 i} / 14 \\
(\mathrm{mmol})\end{array}$ & $\begin{array}{l}\mathrm{CH}_{2} \mathrm{Cl}_{2} \\
(\mathrm{~mL})\end{array}$ & & & \\
\hline $2 \mathrm{~d}(0.52)$ & 12 & 0.60 & $11 \mathrm{i}(0.13)$ & 25 & $15 d$ & 26 & $\mathrm{C}_{70} \mathrm{H}_{52} \mathrm{~N}_{4}(949.2)$ \\
\hline $2 \mathrm{~g}(0.20)$ & 10 & 0.13 & $11 \mathrm{i}(0.10)$ & 25 & $15 \mathrm{~g}$ & 55 & $\mathrm{C}_{70} \mathrm{H}_{52} \mathrm{~N}_{4}(949.2)$ \\
\hline $2 \mathrm{~h}(0.60)$ & 10 & 0.39 & $11 \mathrm{i}(0.10)$ & $25^{\mathrm{c}}$ & $15 \mathrm{~h}$ & 19 & $\mathrm{C}_{72} \mathrm{H}_{56} \mathrm{~N}_{4}(977.2)$ \\
\hline $2 \mathbf{d}(0.09)$ & 5 & 0.06 & $14(0.04)$ & 20 & $16 d$ & $18^{\mathrm{d}}$ & $\mathrm{C}_{80} \mathrm{H}_{64} \mathrm{~N}_{4}(1081.4)$ \\
\hline $2 \mathrm{~g}(0.16)$ & 10 & 0.10 & $14(0.08)$ & 25 & $16 \mathrm{~g}$ & $49^{\mathrm{f}}$ & $\mathrm{C}_{80} \mathrm{H}_{64} \mathrm{~N}_{4}(1081.4)$ \\
\hline $2 \mathrm{~h}(0.48)$ & 10 & 0.30 & $14(0.08)$ & 30 & $16 \mathrm{~h}$ & $18^{\mathrm{f}}$ & $\mathrm{C}_{82} \mathrm{H}_{68} \mathrm{~N}_{4}(1109.4)$ \\
\hline
\end{tabular}

a Alle Produkte (violett-schwarze bis schwarze Feststoffe) zeigten nach der Chromatographie und Umkristallisation der ersten Hauptfraktion aus $\mathrm{CH}_{2} \mathrm{Cl}_{2} / \mathrm{MeOH}$ einen $\mathrm{mp}>270^{\circ} \mathrm{C}$ (unkorrigiert, bestimmt mit einem Büchi SMP 20).

b Alle Verbindungen ergaben korrekte Mikroanalysen $(\mathrm{C} \pm 0.40, \mathrm{H} \pm 0.40)$ oder die miltels Massenfeinbestimmung nach dem Peakmatch-Verfahren erhaltenen Werte $(m / z \pm 0.0009)$.

c 1,2-Dichlorethan als Lösungsmittel.

d Nach Lösen in $\mathrm{CH}_{2} \mathrm{Cl}_{2}$, Ausfällen mit $\mathrm{MeOH}$ und Trocknen i. Hochvak.

e Nach Umkristallisation der 1. Hauptraktion.

f Nach zweimaliger Chromatographie an $\mathrm{SiO}_{2}$ mit $\mathrm{CH}_{2} \mathrm{Cl}_{2}$ als Laufmittel und Umkristallisation aus $\mathrm{CH}_{2} \mathrm{Cl}_{2} / \mathrm{MeOH} / \mathrm{Hexan}(1: 1: 1)$. 
<smiles>CC(C=O)=CC=CC=C(C)C=O</smiles>

10

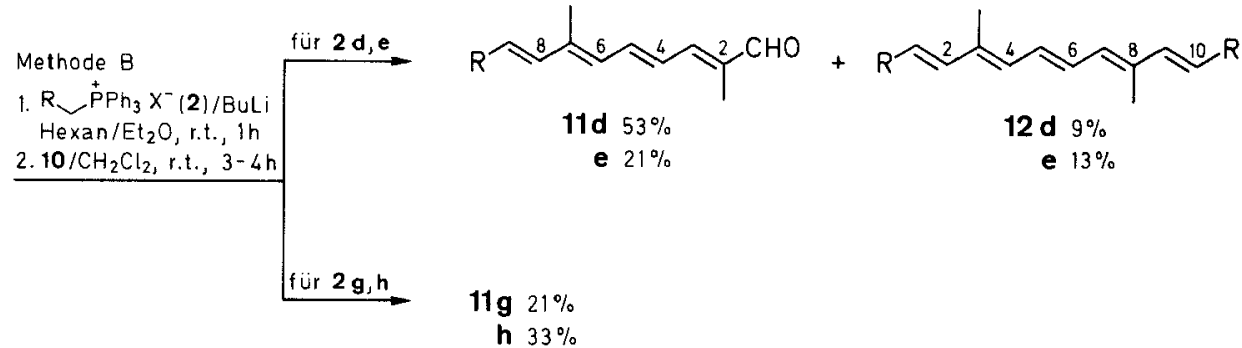

h $33 \%$

Schema C

Methode $C$
1. $2 \mathrm{e} / \mathrm{BuL} / \mathrm{H} / \mathrm{Hexan} / \mathrm{Et}_{2} \mathrm{O}$, r.t., $0.5 \mathrm{~h}$
2. 11d $/ \mathrm{CH}_{2} \mathrm{Cl}_{2}$, r.t., $2 \mathrm{~h}$

$11 d$<smiles>CC(/C=C/c1cccc2ccccc12)=C\C=C\C=C\C(C)=C\c1c2ccccc2cc2ccccc12</smiles>

13
4. Tetraphenylporphyrin(TPP)-substituierte Polyenale 11i, 14 und TPP-Anthryl- substituierte Polyene 15, 16

Aufgrund theoretischer Überlegungen sollte ein Energietransfer über konjugierte Polyene, die endständig einerseits einen Anthryl- bzw. Naphthyl-Rest und andererseits einen Porphyrin-Rest besitzen, besonders begünstigt sein $^{1}$. Da sich die UV/VIS-Absorptions- und Emissionsbanden der jeweiligen Molekülteile (Porphyrin, konjugiertes Polyen und Anthracen) ausreichend stark von einander unterscheiden, sollte auch der Nachweis eines Energietransfers in solchen Verbindungen möglich sein $^{1}$.
Das für den Aufbau der angestrebten Verbindungen erforderliche 2-Formyl-5,10,15,20-tetraphenylporphyrin (TPP-CHO) ist leicht zugänglich ${ }^{25}$, seine Umsetzung mittels Wittig-Olefinierungen $\mathrm{zu}$ Vinyl-substituierten TPP-Derivaten wurde bereits beschrieben ${ }^{26}$. Bei der Umsetzung von TPP-CHO mit dem Ylid des in situ erzeugten Phosphonium-Salzes $6 \mathbf{a}$ (siehe oben) erhielten wir nach Aufarbeitung über Kieselgel und Umkristallisation das TPP-substituierte Tetraenal $11 \mathbf{i}$ in $59 \%$ Ausbeute. Eine Verlängerung der Polyenkette des Tetraenals 11i zum TPP-substituierten Octaenal 14 wurde durch erneute Umsetzung von 11 i mit 6 erreicht. Die HPLC-Analyse<smiles>COC(/C=C/C=C/C=C/C=C(\C)C[PbH](Cl)CCl)OC</smiles>

6 a

$$
\begin{aligned}
& \text { 1. } \mathrm{NaOMe} / \mathrm{MeOH} \\
& \text { 2. } \mathrm{TPP}-\mathrm{CHO}_{\mathrm{CH}} \mathrm{Cl}_{2} \text {, r.t., } 17 \mathrm{~h} \\
& \frac{\mathrm{SiO}_{2} / \mathrm{CH}_{2} \mathrm{Cl}_{2}}{59 \%}
\end{aligned}
$$<smiles>CC(C=O)=CC=CC=C(C)C=C[PH+]</smiles>

$11 \mathrm{i}$

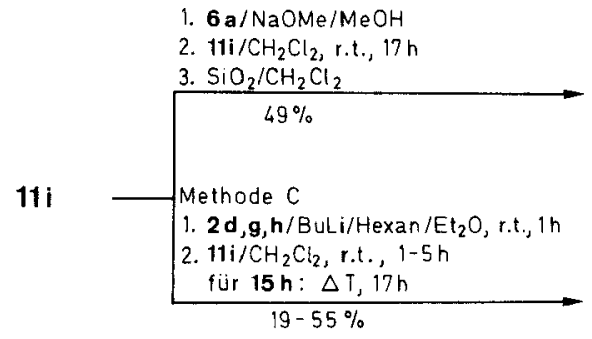<smiles>CC(C=O)=CC=CC=C(C)C=CC(C)=CC=CC=C(C)C=CC=[Pt]</smiles><smiles>[R]C=CC(C)=CC=CC=C(C)C=CC(C)C</smiles>

$15 d, g, h$

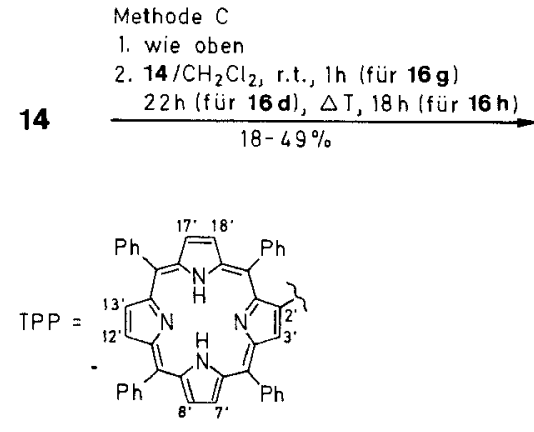<smiles>[R]C=CC(C)=CC=CC=C(C)C=CC(C)=CC=CC=C(C)C=CPC(C)C</smiles>

$16 d, g, h$ 
des hierbei erhaltenen Rohproduktes zeigte das Vorliegen eines Gemisches aus 11i und 14 im Verhältnis von 70:30, durch Umkristallisation aus Dichlormethan/Hexan konnte das Octaenal 14 auf einen Anteil von $99 \%$ bei einer Gesamtausbeute von $49 \%$ (s. experimenteller Teil) angereichert werden (Schema D).

Die TPP-substituierten Pentaene 15 d, g, h und Nonaene 16d, $\mathbf{g}, \mathbf{h}$ wurden schließlich durch Olefinierung des Tetraenals 11i bzw. des Octaenals 14 jeweils mit den Phosphonium-Salzen 2d, g, h erhalten (Schema D, Tabelle 6). Die Reaktionen mit dem sterisch besonders anspuchsvollen Phosphoniumsalz $\mathbf{2 h}$ erforderten drastischere Reaktionsbedingungen und ergaben selbst unter Einsatz eines 6- bis 8-fachen Überschusses an $2 \mathrm{~h}$ und 17-18-stündiger Umsetzung unter Rückfluß nur in schlechten Ausbeuten die entsprechenden Olefine 15h und 16h (Tabelle 6). Exemplarische ${ }^{1} \mathrm{H}-\mathrm{NMR}$-spektroskopische Daten der in diesem Kapitel beschriebenen Polyene 15 und 16 sind in Tabelle 8 mit aufgeführt.

\section{Isomerenverteilung}

Obwohl das eingesetzte Phosphonium-Salz 6 nur $70 \%$ all-E-Anteil aufwies (siehe oben), haben wir die TPPsubstituierten Polyenale $11 \mathrm{i}$ und 14 überwiegend als all$E$-Isomere erhalten; nach zweimaliger Chromatographie und zweimaliger Umkristallisation lagen sie als praktisch reine all-E-Isomere vor (Tabelle 7).

Tabelle 7. Prozentuale Isomerenverteilung der Verbindungen 11d, e, g, h, i, 12d, e, 13, 14, 15d, g, h und 16d, g, h

\begin{tabular}{|c|c|c|}
\hline \multirow{2}{*}{$\begin{array}{l}\text { Ver- } \\
\text { bin- } \\
\text { dung }\end{array}$} & \multicolumn{2}{|c|}{ Isomerenverteilung $(\%)^{\mathrm{a}}\left[\right.$ cis-Peak $\left./ \mathrm{S}_{0} \rightarrow \mathrm{S}_{2}, \lambda_{\max }(\mathrm{nm})\right]$} \\
\hline & all-E-Isomere & $E / Z$-Isomere \\
\hline 11d & $\begin{array}{l}92^{b}, 99^{c}, 100^{c . d} \\
{[-/ 406]}\end{array}$ & $6^{\mathrm{b}}, 1^{\mathrm{c}}[-/ 338], 2^{\mathrm{b}}[-/ 402]$ \\
\hline $11 \mathrm{e}$ & $\begin{array}{l}97^{c}, 100^{c, d} \\
{[-/ 390]}\end{array}$ & $1^{\mathrm{c}}[-/ 382], 2^{\mathrm{c}}[-/ 383]$ \\
\hline $11 \mathrm{~g}$ & $\begin{array}{l}78^{\mathrm{e}}, 97^{\mathrm{e}}, 100^{\mathrm{e}, \mathrm{d}} \\
{[-1408]}\end{array}$ & 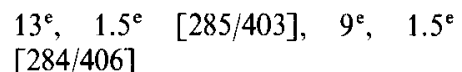 \\
\hline $11 \mathrm{~h}$ & $\begin{array}{l}100^{c}, 100^{c, d} \\
{[-/ 397]}\end{array}$ & \\
\hline $11 \mathrm{i}$ & $\begin{array}{l}89^{\mathrm{c}}, 98^{\mathrm{e}}, 100^{\mathrm{d}} \\
{[\mathrm{f} / \approx 454]}\end{array}$ & $8^{\mathrm{c}}, 1^{\mathrm{e}}[\mathrm{f} / \approx 448], 3^{\mathrm{c}}, 1^{\mathrm{e}}[\mathrm{f} / \approx 448]$ \\
\hline 12d & $\begin{array}{l}100^{c}, 100^{\mathrm{c}, \mathrm{d}} \\
{[-/ 426]}\end{array}$ & \\
\hline $12 \mathrm{e}$ & $\begin{array}{l}99^{c}, 100^{c}, 100^{c, d} \\
{[-/ 415]}\end{array}$ & $1^{\mathrm{c}}$ \\
\hline 13 & $\begin{array}{l}100^{\mathrm{c}}, 100^{\mathrm{c}, \mathrm{d}} \\
{[-/ 420]}\end{array}$ & \\
\hline 14 & $\begin{array}{l}99^{\circ}, 100^{\mathrm{c} . \mathrm{d}} \\
{[\mathrm{f} / 491]}\end{array}$ & $--^{\mathfrak{c}}$ \\
\hline 15d & $\begin{array}{l}95^{\mathrm{e}}, 100^{\mathrm{e}, \mathrm{d}} \\
{[\mathrm{f} / \approx 459]}\end{array}$ & $\begin{array}{l}3^{\mathrm{e}} \quad[\mathrm{f} / \approx 455], \quad 1^{\mathrm{e}} \quad[\mathrm{f} / \approx 455], \\
{[\mathrm{l} / \approx 454]}\end{array}$ \\
\hline $15 \mathrm{~g}$ & $\begin{array}{l}96^{\mathrm{e}}, 100^{\mathrm{c} \cdot \mathrm{d}} \\
{[\mathrm{f} / \approx 470]}\end{array}$ & $4^{c}[\mathrm{f} / \approx 466]$ \\
\hline 15h & $\begin{array}{l}86^{b}, 100^{\mathrm{c}}, 100^{\mathrm{c}, \mathrm{d}} \\
{[\mathrm{f} / \approx 458]}\end{array}$ & $\begin{array}{l}3^{\mathrm{b}} \quad[\mathrm{f} / \approx 453], \quad 2^{\mathrm{b}} \quad[\mathrm{f} / \approx 452], \\
{[\mathrm{f} / \approx 451]}\end{array}$ \\
\hline $16 \mathrm{~d}$ & $84^{\mathrm{c}, \mathrm{d}}$ & $16^{\mathrm{c}, \mathrm{d}}$ \\
\hline $16 \mathrm{~g}$ & $83^{e, d}$ & $17^{\mathrm{e}, \mathrm{d}}$ \\
\hline 16h & $87^{\mathrm{c}, \mathrm{d}}$ & $13^{\mathrm{c}, \mathrm{d}}$ \\
\hline
\end{tabular}

a-e Siehe Tabelle 2.

f Mögliche cis-Peaks sind von der intensiven Soret-Bande der TPP-Endgruppe überlagert.
Von den erhaltenen Pentaenen 15 und Nonaenen 16 ließen sich nur die Verbindungen 15 gut mittels HPLC auftrennen, sie enthielten nach Umkristallisation zu $95-100 \%$ das all-E-Isomere (Tabelle 7). Die ${ }^{1} \mathrm{H}-\mathrm{NMR}$ spektroskopisch bestimmte Isomerenverteilung der dargestellten Nonaene 16 ergab einen all-E-Isomerenanteil der gereinigten Verbindungen 16d, $\mathbf{g}$, h von $83-87 \%$ (Tabelle 7).

Sämtliche Lösungsmittel wurden gereinigt und getrocknet. Die Umsetzungen wurden in ausgeheizten und mit Reinstickstoff belüfteten Apparaturen unter Feuchtigkeitsauschluß und Schutzgasatmosphäre durchgeführt. Die Reaktionskolben waren stets mit einem Gummiseptum verschlossen, durch das mittels einer Nadel ein schwacher $\mathrm{N}_{2}$-Strom durch die Apparatur geleitet wurde.

\section{Tetramethylheptadecaoctaenale 3, Tetramethyloctadecanonaene} 4,5, 2,7-Dimethylnonatetraenale 11 und 3,8-Dimethyldecapentaene 12, 13; allgemeine Arbeitsvorschriften:

Methode A: Die Reaktionen werden in einem Dreihalskolben mit Tropftrichter (mit aufgesetztem, um ca. $90^{\circ}$ abgewinkeltem Glasrohr, das mit einem zur Ylid-Erzeugung dienenden Zweihalskolben versehen ist) und Rückflußkühler (mit Blasenzähler und SicapentTrockenrohr) unter $\mathrm{N}_{2}$ durchgeführt. Bei schräggestellter Apparatur wird das Phosphoniumsalz 2 im Zweihalskolben in $\mathrm{Et}_{2} \mathrm{O}$ suspendiert, der Kolben mit einem Gummiseptum verschlossen, eine 1.6 M BuLi-Lösung in Hexan in den Zweihalskolben gespritzt und bei r.t. gerührt. Nach Zugabe von $\mathrm{CH}_{2} \mathrm{Cl}_{2}(1-2 \mathrm{~mL}$, zur Zerstörung von überschüssigem $\mathrm{BuLi}$ ) wird die Apparatur aufgerichtet (damit die Ylid-Lösung über das Glasrohr in den Tropftrichter fließen kann), die Lösung des Polyendialdehyds 1 bzw. 10 in $\mathrm{CH}_{2} \mathrm{Cl}_{2}$ durch das Gummiseptum in den Dreihalskolben gespritzt, dann die Ylid-Lösung aus dem Tropfrichter innerhalb $2 \mathrm{~h}$ zugetropft und die Reaktionslösung bei r.t. gerührt (unter Rückfluß für die Substrate 2a, c). Die Reaktion wird dünnschichtchromatographisch auf Kieselgel mit $\mathrm{CH}_{2} \mathrm{Cl}_{2}$ als Laufmittel verfolgt. Das Gemisch wird auf $\mathrm{H}_{2} \mathrm{O}$ gegossen und mit $\mathrm{CH}_{2} \mathrm{Cl}_{2}$ ausgeschüttelt, die organischen Phasen werden mit $\mathrm{H}_{2} \mathrm{O}$ gewaschen, getrocknet $\left(\mathrm{Na}_{2} \mathrm{SO}_{4}\right)$ und filtriert. Das Filtrat wird eingeengt und über eine Kieselgelsäule $(45 \times 5 \mathrm{~cm})$ mit $\mathrm{CH}_{2} \mathrm{Cl}_{2}$ als Laufmittel chromatographiert. Die Hauptfraktionen werden gesammelt, eingeengt und im Hochvakuum getrocknet; die 1. Hauptfraktion enthält die Diolefinierungsprodukte 4 und 12, die 2. Hauptfraktion die Monoolefinierungsprodukte 3 und $\mathbf{1 1}$.

Methode B: Herstellung der Verbindungen wie bei Methode A beschrieben. Das Gemisch wird am Rotationsverdampfer eingeengt und wie bei Methode A chromatographiert.

Aus der jeweils erhaltenen ersten Hauptfraktion (ein Gemisch aus 2 Verbindungen) werden nach Lösen in $\mathrm{CH}_{2} \mathrm{Cl}_{2}$ durch Zutropfen von $\mathrm{MeOH}$ die Nonaene 4 ausgefällt, abfiltriert, mit $\mathrm{MeOH}$ gewaschen und im Hochvakuum getrocknet.

Methode C: In einen Zweihalskolben mit Rückflußkühler, Blasenzähler und Sicapent-Trockenrohr wird unter $\mathrm{N}_{2}$ zu der Suspension von 2 in $\mathrm{Et}_{2} \mathrm{O}$ eine 1.6 M BuLi-Lösung in Hexan gespritzt und bei r.t. gerührt. Nach Zugabe von $1 \mathrm{~mL} \mathrm{CH} \mathrm{Cl}_{2}$ wird die Lösung des entsprechenden Polyenaldehyds oder Polyendialdehyds in $\mathrm{CH}_{2} \mathrm{Cl}_{2}$ zugespritzt und bei r.t. gerührt. Die Reaktion wird wie bei Methode A, B dünnschichtchromatographisch verfolgt. Zur Aufarbeitung wird das Gemisch am Rotationsverdampfer eingeengt und wie unter Methode A beschrieben über eine Kieselgelsäule chromatographiert.

Hexamethylpentacosadodecaenale $7 \mathrm{a}-\mathrm{e}$; allgemeine Arbeitsvorschrift:

In einem Zweihalskolben mit Rückflußkühler, Blasenzähler und Sicapent-Trockenrohr wird unter $\mathrm{N}_{2}$ zu der Lösung des aus 8Hydroxy-2,7-dimethyloctatrienal hergestellten Phosphonium-Salzes $6^{23}$ in $\mathrm{MeOH}$ Orthoameisensäure-trimethylester, anschlieBend cine Lösung von $p$-Toluolsulfonsäure $\mathrm{TsOH}$, in $\mathrm{MeOH}$ (ca. $5 \mathrm{~mL}$ ) zugespritzt und $2 \mathrm{~h}$ bei r.t. gerührt. Nach Zugeben 
von Pyridin (zum Abfangen der Säure) wird die angegebene Menge einer aus $\mathrm{Na}(0.23 \mathrm{~g})$ und $\mathrm{MeOH}(10 \mathrm{~mL})$ frisch bereiteten $1 \mathrm{~N} \mathrm{NaOMe-Lösung,} \mathrm{dann} \mathrm{die} \mathrm{Lösung} \mathrm{des} \mathrm{Polyenaldehyds}$ in $\mathrm{CH}_{2} \mathrm{Cl}_{2}$ zugespritzt und die angegebene Zeit bei r.t. gerührt. Die Reaktion wurde dünnschichtchromatographisch an $\mathrm{Al}_{2} \mathrm{O}_{3}$ mit $\mathrm{CH}_{2} \mathrm{Cl}_{2}$ als Laufmittel verfolgt. Zur Aufarbeitung wurde das Reaktionsgemisch am Rotationsverdampfer eingeengt und wie unter Methode A über eine Kieselgelsäule chromatographiert.
Hexamethylhexacosatridecaene 8d, e, 9 und TPP-Anthryl-substituierte Polyene 15d, g, h:

Diese Verbindungen werden ebenfalls nach oben beschriebener Methode $C$ hergestellt.

\section{2,7-Dimethyl-9- $\left[2^{\prime}-\left(5^{\prime}, 10^{\prime}, 15^{\prime}, 20^{\prime}\right.\right.$-tetraphenylporphyrinyl) $]-$} nonatetraenal (11i):

Wie für Verbindungen $7 \mathrm{a}-\mathrm{c}$ beschrieben aus $1.5 \mathrm{mmol} 6$ in $\mathrm{MeOH}$ $(15 \mathrm{~mL})$, Orthoameisensäure-trimethylester $(3.0 \mathrm{mmol}), \mathrm{TsOH}$ $(2 \mathrm{mg})$ in $\mathrm{MeOH}(1 \mathrm{~mL})$, Pyridin $(0.25 \mathrm{~mL}), 1 \mathrm{~N} \mathrm{NaOMe}-L$ ösung

Tabelle 8. Exemplarische ${ }^{1} \mathrm{H}-\mathrm{NMR}$-Spektroskopische Daten ${ }^{\mathrm{a}}$ der hergestellten Verbindungen

\begin{tabular}{|c|c|c|}
\hline $\begin{array}{l}\text { Ver- } \\
\text { bindung }\end{array}$ & Isomere & $\begin{array}{l}{ }^{1} \mathrm{H}-\mathrm{NMR}\left(\mathrm{CDCl}_{3} / \mathrm{TMS}\right) \\
\delta, J(\mathrm{~Hz})\end{array}$ \\
\hline 3a & all-E & $\begin{array}{l}1.909\left(\mathrm{~d}, 2-\mathrm{CH}_{3}\right), 2.024\left(2 \mathrm{~s}, 6-\mathrm{CH}_{3}, 11-\mathrm{CH}_{3}\right), 2.070\left(\mathrm{~s}, 15-\mathrm{CH}_{3}\right), 6.355\left(\mathrm{~d}, J_{9,10}=10.9,10-\mathrm{H}\right), 6.452\left(\mathrm{~d}, J_{7,8}\right. \\
=10.15,7-\mathrm{H}), 6.452\left(\mathrm{~d}, J_{13,14}=10.15,14-\mathrm{H}\right), 6.493\left(\mathrm{~d}, J_{12,13}=14.4,12-\mathrm{H}\right), 6.616\left(\mathrm{~d}, J_{16,17}=15.9,16-\mathrm{H}\right), \\
6.672-6.827(\mathrm{~m}, 4-, 5-, 8-, 9-, 13-\mathrm{H}), 6.947\left(\mathrm{~d}, J_{3,4}=9.4,3-\mathrm{H}\right), 7.043(\mathrm{~d}, 17-\mathrm{H}), 7.512-7.556\left(\mathrm{~m}, 2^{\prime}-, 6^{\prime}-\mathrm{H}\right), 8.156- \\
8.200\left(\mathrm{~m}, 3^{\prime}-, 5^{\prime}-\mathrm{H}\right), 9.461(\mathrm{~s}, \mathrm{CHO})\end{array}$ \\
\hline \multirow[t]{2}{*}{$4 \mathbf{a}$} & $\begin{array}{l}E / Z \\
\text { all-E }\end{array}$ & $\begin{array}{l}7.579-7.664\left(\mathrm{~m}, 2^{\prime}-, 6^{\prime}-\mathrm{H}\right), 8.229-8.321\left(\mathrm{~m}, 3^{\prime}-, 5^{\prime}-\mathrm{H}\right) \\
2.011\left(\mathrm{~s}, 7-, 12-\mathrm{CH}_{3}\right), 2.065\left(\mathrm{~s}, 3-, 16-\mathrm{CH}_{3}\right), 6.343(\mathrm{mc}, 8-, 11-\mathrm{H}), 6.451\left(\mathrm{~d}, J_{4,5 ; 14 ; 15}=10.9,4-, 15-\mathrm{H}\right), 6.493(\mathrm{~d}, \\
\left.J_{5,6 ; 13,14}=14.1,6-, 13-\mathrm{H}\right), 6.601\left(\mathrm{~d}, J_{1,2 ; 17.18}=15.9,2-17-\mathrm{H}\right), 6.600-6.738(\mathrm{~m}, 5-, 9-, 10-, 14-\mathrm{H}), 7.045(\mathrm{~d}, \\
\left.J_{1,2: 17,18}=15.9,1-, 18-\mathrm{H}\right), 7.516-7.545\left(\mathrm{~m}, 2^{\prime}-, 6^{\prime}-, 2^{\prime \prime}-6^{\prime \prime}-\mathrm{H}\right), 8.161-8.191\left(\mathrm{~m}, 3^{\prime}-, 5^{\prime}-, 3^{\prime \prime}-, 5^{\prime \prime}-\mathrm{H}\right)\end{array}$ \\
\hline & $E / Z$ & $\begin{array}{l}2.033,2.049\left(2 \mathrm{~s}, 3-, 7-, 12-, 16-\mathrm{CH}_{3}\right), 6.208\left(\mathrm{~d}, J=12.2,2 \mathrm{H}_{\text {olefin }}\right), 6.813-6.931\left(\mathrm{~m}, 4 \mathrm{H}_{\mathrm{olefin}}\right), 7.026\left(\mathrm{~d}, J_{1,2 ; 17.18}\right. \\
=14.45,1-, 18-\mathrm{H}), 7.557-7.606\left(\mathrm{~m}, 2^{\prime}-, 6^{\prime}-, 2^{\prime \prime}-6^{\prime \prime}-\mathrm{H}\right), 8.204-8.220\left(\mathrm{~m}, 3^{\prime}-, 5^{\prime}-, 3^{\prime \prime}-, 5^{\prime \prime}-\mathrm{H}\right)\end{array}$ \\
\hline \multirow[t]{2}{*}{5} & all-E & $\begin{array}{l}2.014\left(\mathrm{~s}, 12-\mathrm{CH}_{3}\right), 2.040\left(\mathrm{~s}, 7-\mathrm{CH}_{3}\right), 2.176\left(\mathrm{~s}, 16-\mathrm{CH}_{3}\right), 2.304\left(\mathrm{~s}, 3-\mathrm{CH}_{3}\right), 6.302-6.477(\mathrm{~m}, 4-, 6-, 8-, 11-, 13-, 15-\mathrm{H}), \\
6.663-6.816(\mathrm{~m}, 2-, 5-, 9-, 10-, 14-\mathrm{H}), 6.975\left(\mathrm{~d}, J_{17.18}=15.6,17-\mathrm{H}\right), 7.343-7.583\left(\mathrm{~m}, 1-, 18-, 2^{\prime}-, 3^{\prime}-, 6^{\prime}-, 7^{\prime}-, 3^{\prime \prime}-,\right. \\
\left.6^{\prime \prime}-7^{\prime \prime}-\mathrm{H}\right), 7.686-7.710\left(\mathrm{~m}, 2^{\prime \prime}-\mathrm{H}\right), 7.742-7.769\left(\mathrm{~m}, 4^{\prime \prime}-\mathrm{H}\right), 7.835-7.867\left(\mathrm{~m}, 5^{\prime \prime}-\mathrm{H}\right), 7.986-8.019\left(\mathrm{~m}, 4^{\prime}-, 5^{\prime}-\mathrm{H}\right), \\
8.179-8.210\left(\mathrm{~m}, 8^{\prime \prime}-\mathrm{H}\right), 8.299-8.333\left(\mathrm{~m}, 1^{\prime}-, 8^{\prime}-\mathrm{H}\right), 8.370\left(\mathrm{~s}, 10^{\prime}-\mathrm{H}\right)\end{array}$ \\
\hline & $E / Z$ & $2.060\left(\mathrm{~d}, 7-\mathrm{CH}_{3}\right), 6.178\left(\mathrm{~d},{ }^{3} \mathrm{~J}=13.2,2 \mathrm{H}_{\text {olefin }}\right)$ \\
\hline \multirow[t]{2}{*}{$7 \mathbf{a}$} & all-E & $\begin{array}{l}1.889\left(\mathrm{~s}, 2-\mathrm{CH}_{3}\right), 2.008\left(2 \mathrm{~s}, 10-, 14-\mathrm{CH}_{3}\right), 2.037\left(\mathrm{~s}, 7-\mathrm{CH}_{3}\right), 2.049\left(\mathrm{~s}, 19-\mathrm{CH}_{3}\right), 2.062\left(\mathrm{~s}, 23-\mathrm{CH}_{3}\right), 6.333-6.727(\mathrm{~m}, \\
4-, 6-, 8-, 9-, 11-\text { bis } 13-, 15-\text { bis } 18-, 20-\text { bis } 22-, 24-\mathrm{H}), 6.969\left(\mathrm{~d}, J_{3,4}=11.4,3-\mathrm{H}\right), 7.042\left(\mathrm{~d}, J_{24.25}=15.9,25-\mathrm{H}\right), \\
7.512-7.542\left(\mathrm{~m}, 2^{\prime}-, 6^{\prime}-\mathrm{H}\right), 8.159-8.188\left(\mathrm{~m}, 3^{\prime}-, 5^{\prime}-\mathrm{H}\right), 9.458(\mathrm{~s}, \mathrm{CHO})\end{array}$ \\
\hline & $E / Z$ & $6.182\left(\mathrm{~d},{ }^{3} J=9.9, \mathrm{H}_{\text {olefin }}\right), 9.464(\mathrm{~s}, \mathrm{CHO})$ \\
\hline \multirow[t]{2}{*}{$8 d$} & all-E & $\begin{array}{l}1.975\left(\mathrm{~s}, 16-\mathrm{CH}_{3}\right), 1.988\left(\mathrm{~s}, 12-\mathrm{CH}_{3}\right), 2.000\left(\mathrm{~s}, 19-\mathrm{CH}_{3}\right), 2.032\left(\mathrm{~s}, 7-\mathrm{CH}_{3}\right), 2.283\left(\mathrm{~s}, 24-\mathrm{CH}_{3}\right), 2.300\left(\mathrm{~s}, 3-\mathrm{CH}_{3}\right), \\
6.212-6.878(\mathrm{~m}, 2-, 4-\text { bis } 6-, 8-, 9-\text { bis } 11-, 13-\text { bis } 15-, 17-, 18-, 20-\mathrm{bis} 23-, 25-\mathrm{H}), 7.390-7.494\left(\mathrm{~m}, 1-, 26-, 2^{\prime}-, 3^{\prime}-\text {, }\right. \\
\left.6^{\prime}-, 7^{\prime}-, 2^{\prime \prime}-, 3^{\prime \prime}-, 6^{\prime \prime}-, 7^{\prime \prime}-\mathrm{H}\right), 7.983-8.015\left(\mathrm{~m}, 4^{\prime}-, 5^{\prime}-, 4^{\prime \prime}-, 5^{\prime \prime}-\mathrm{H}\right), 8.297-8.329\left(\mathrm{~m}, 1^{\prime}-, 8^{\prime}-, 1^{\prime \prime}-, 8^{\prime \prime}-\mathrm{H}\right), 8.376\left(\mathrm{~s}, 10^{\prime}-,\right. \\
\left.10^{\prime \prime}-\mathrm{H}\right)\end{array}$ \\
\hline & $E / Z$ & $6.106-6.212\left(\mathrm{~m}, 2 \mathrm{H}_{\text {olef in }}\right), 6.878-7.027\left(\mathrm{~m}, 2 \mathrm{H}_{\text {olef in }}\right)$ \\
\hline \multirow[t]{2}{*}{$9^{b}$} & all-E & $\begin{array}{l}1.990\left(\mathrm{~s}, 12-, 16-, 19-\mathrm{CH}_{3}\right), 2.034\left(\mathrm{~s}, 7-\mathrm{CH}_{3}\right), 2.156\left(\mathrm{~s}, 24-\mathrm{CH}_{3}\right), 2.301\left(\mathrm{~s}, 3-\mathrm{CH}_{3}\right), 6.282-6.809(\mathrm{~m}, 2-, 4-\text { bis } 6-, \\
\text { 9- }\end{array}$ \\
\hline & $E / Z$ & $6.086-6.200\left(\mathrm{~m}, 2 \mathrm{H}_{\text {olef in }}\right)$ \\
\hline $11 d$ & all-E & $\begin{array}{l}1.893\left(\mathrm{~d},{ }^{4} J=0.67,2-\mathrm{CH}_{3}\right), 2.351\left(\mathrm{~s}, 7-\mathrm{CH}_{3}\right), 6.415(\mathrm{~d}, 6-\mathrm{H}), 6.752\left(\mathrm{dd}, J_{4,5}=14.45,4-\mathrm{H}\right), 6.768\left(\mathrm{~d}, J_{8,9}=16.2,\right. \\
8-\mathrm{H}), 7.009\left(\mathrm{~d}, J_{3,4}=11.4,3-\mathrm{H}\right), 7.151\left(\mathrm{dd}, J_{5,6}=11.7,5-\mathrm{H}\right), 7.449-7.509\left(\mathrm{~m}, 2^{\prime}-, 3^{\prime}-, 6^{\prime}-, 7^{\prime}-\mathrm{H}\right), 7.591(\mathrm{~d}, 9-\mathrm{H}), \\
7.995-8.039\left(\mathrm{~m}, 4^{\prime}-, 5^{\prime}-\mathrm{H}\right), 8.247-8.300\left(\mathrm{~m}, \mathrm{I}^{\prime}-, 8^{\prime}-\mathrm{H}\right), 8.397\left(\mathrm{~s}, 10^{\prime}-\mathrm{H}\right), 9.496(\mathrm{~s}, \mathrm{CHO})\end{array}$ \\
\hline $12 d$ & all-E & $\begin{array}{l}2.290\left(\mathrm{~s}, 3-, 8-\mathrm{CH}_{3}\right), 6.418\left(\mathrm{~d}, J_{4,5 ; 6,7}=9.8,4-, 7-\mathrm{H}\right), 6.759\left(\mathrm{~d}, J_{1,2 ; 9.10}=16.1,2-, 9-\mathrm{H}\right), 6.821(\mathrm{mc}, 5-, 6-\mathrm{H}), \\
7.420-7.502\left(\mathrm{~m}, 1-, 10-, 2^{\prime}-, 3^{\prime}-, 6^{\prime}-, 7^{\prime}-, 2^{\prime \prime}-, 3^{\prime \prime}-, 6^{\prime \prime}-, 7^{\prime \prime}-\mathrm{H}\right), 7.985-8.037\left(\mathrm{~m}, 4^{\prime}-, 5^{\prime}-, 4^{\prime \prime}-, 5^{\prime \prime}-\mathrm{H}\right), 8.295-8.347(\mathrm{~m}, \\
\left.1^{\prime}-, 8^{\prime}-, 1^{\prime \prime}-, 8^{\prime \prime}-\mathrm{H}\right), 8.318\left(\mathrm{~s}, 10^{\prime}-, 10^{\prime \prime}-\mathrm{H}\right)\end{array}$ \\
\hline $13^{b}$ & all-E & $\begin{array}{l}2.163\left(\mathrm{~s}, 8-\mathrm{CH}_{3}\right), 2.306\left(\mathrm{~s}, 3-\mathrm{CH}_{3}\right), 6.401\left(\mathrm{~d}, J_{4,5}=10.1,4-\mathrm{H}\right), 6.470\left(\mathrm{~d}, J_{6.7}=9.9,7-\mathrm{H}\right), 6.752\left(\mathrm{~d}, J_{1,2}=15.9,2-\right. \\
\mathrm{H}), 6.773-6.830(\mathrm{~m}, 5-, 6-\mathrm{H}), 6.986\left(\mathrm{~d}, J_{9,10}=15.6,9-\mathrm{H}\right), 7.369-7.563\left(\mathrm{~m}, 1-, 10-, 2^{\prime}-, 3^{\prime}-, 6^{\prime}-, 7^{\prime}-3^{\prime \prime}-, 6^{\prime \prime}-, 7^{\prime \prime}-\mathrm{H}\right), \\
7.698-7.722\left(\mathrm{~m}, 2^{\prime \prime}-\mathrm{H}\right), 7.757-7.785\left(\mathrm{~m}, 4^{\prime \prime}-\mathrm{H}\right), 7.844-7.875\left(\mathrm{~m}, 5^{\prime \prime}-\mathrm{H}\right), 7.984-8.037\left(\mathrm{~m}, 4^{\prime}-, 5^{\prime}-\mathrm{H}\right), 8.180-8.210 \\
\left(\mathrm{~m}, 8^{\prime \prime}-\mathrm{H}\right), 8.292-8.347\left(\mathrm{~m}, 1^{\prime}-, 8^{\prime}-\mathrm{H}\right), 8.380\left(\mathrm{~s}, 10^{\prime}-\mathrm{H}\right)\end{array}$ \\
\hline $15 d^{c}$ & all-E & 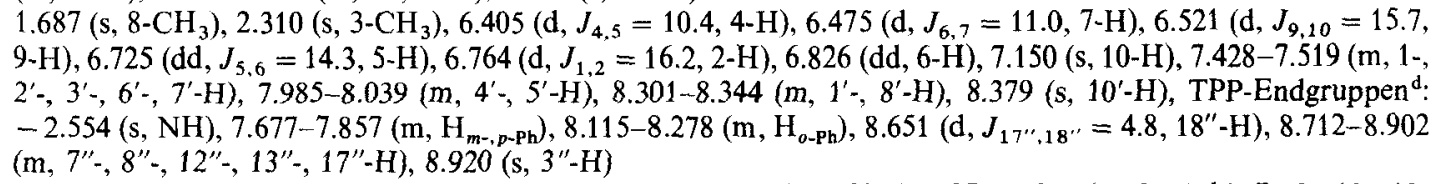 \\
\hline \multirow[t]{2}{*}{$16 d^{e}$} & all-E & $\begin{array}{l}1.682\left(\mathrm{~s}, 16-\mathrm{CH}_{3}\right), 1.993\left(\mathrm{~s}, 11-\mathrm{CH}_{3}\right), 2.008\left(\mathrm{~s}, 8-\mathrm{CH}_{3}\right), 2.293\left(\mathrm{~s}, 3-\mathrm{CH}_{3}\right), 6.272-6.817(\mathrm{~m}, 2-, 4-\text { bis } 7-, 9-, 10-, 12- \\
\text { bis } 15-, 17-\mathrm{H}), 7.134\left(\mathrm{~d}, J_{17.18}=15.4,18-\mathrm{H}\right), 7.407-7.513\left(\mathrm{~m}, 1-, 2^{\prime}-, 3^{\prime}-, 6^{\prime}-, 7^{\prime}-\mathrm{H}\right), 7.970-8.019\left(\mathrm{~m}, 4^{\prime}-, 5^{\prime}-\mathrm{H}\right) \text {, } \\
8.300-8.334\left(\mathrm{~m}, 1^{\prime}-, 8^{\prime}-\mathrm{H}\right), 8.372\left(\mathrm{~s}, 10^{\prime}-\mathrm{H}\right) \\
\text { TPP-Endgruppe }{ }^{\mathrm{d}}:-2.553(\mathrm{~s}, \mathrm{NH}), 7.675-7.879\left(\mathrm{~m}, \mathrm{H}_{m ; \mathrm{p}-\mathrm{Ph}}\right), 8.111-8.242\left(\mathrm{~m}, \mathrm{H}_{o-\mathrm{ph}}\right), 8.646\left(\mathrm{~d}, J_{17^{\prime \prime}, 18^{\prime \prime}}=4.8 \text {, }\right. \\
\left.18^{\prime \prime}-\mathrm{H}\right), 8.723-8.812\left(\mathrm{~m}, 7^{\prime \prime}-, 8^{\prime \prime}-, 12^{\prime \prime}-, 13^{\prime \prime}-, 17^{\prime \prime}-\mathrm{H}\right), 8.902\left(\mathrm{~s}, 3^{\prime \prime}-\mathrm{H}\right)\end{array}$ \\
\hline & $E / Z$ & $2.320\left(\mathrm{~s}, 3-\mathrm{CH}_{3}\right), 6.145-6.272\left(\mathrm{~m}, \mathrm{H}_{\text {olefin }}\right), 6.817-7.025\left(\mathrm{~m}, \mathrm{H}_{\text {olefin }}\right), 8.397\left(\mathrm{~s}, 10^{\prime}-\mathrm{H}\right), 8.936\left(\mathrm{~s}, 3^{\prime \prime}-\mathrm{H}\right)$ \\
\hline
\end{tabular}

\footnotetext{
a Gemessen bei $300 \mathrm{MHz}$ mit einem Bruker CXP-300 Spektrometer.

b Bezifferung analog Verbindung $\mathbf{5}$.

- Noch zweifacher Chromatographie und Umfällen bzw. Umkristallisation.

¿ Bezifferung der TPP-Endgruppe: ,analog“.

' Nach Chromatographie und Umfällen bzw. Umkristallisation.
} 
in $\mathrm{MeOH}(1.5 \mathrm{~mL}), 2$-Formyl-5,10,15,20-tetraphenylporphyrin 25 (TPP-CHO, $500 \mathrm{mg}$ ) in $\mathrm{CH}_{2} \mathrm{Cl}_{2}(125 \mathrm{~mL}$ ) nach $17 \mathrm{~h}$ Rühren bei r.t. Die Hauptfraktion wurde aus $\mathrm{CH}_{2} \mathrm{Cl}_{2} / \mathrm{Hexan}$ umkristallisiert. Ausbeute: $59 \% 11 \mathrm{i}$ als blauvioletter Feststoff, $\mathrm{mp}>270^{\circ} \mathrm{C}$.

$\mathrm{C}_{55} \mathrm{H}_{42} \mathrm{~N}_{4} \mathrm{O}(775.0)$

Nach Chromatographie an $\mathrm{SiO}_{2}$ mit $\mathrm{CH}_{2} \mathrm{Cl}_{2}$ als Laufmittel und Umkristallisation aus $\mathrm{CH}_{2} \mathrm{Cl}_{2} / \mathrm{Hexan}$ Ausbeute: $43 \% 11 \mathrm{i}$ als blauviolette Kristalle.

${ }^{1} \mathrm{H}-\mathrm{NMR}\left(300 \mathrm{MHz}, \mathrm{CDCl}_{3} / \mathrm{TMS}\right)$ : all- $E$-isomer $\delta=1.742(\mathrm{~s}, 3 \mathrm{H}$, 7- $\left.\mathrm{CH}_{3}\right), 1.912\left(\mathrm{~s}, 3 \mathrm{H}, 2-\mathrm{CH}_{3}\right), 6.464\left(\mathrm{~d},{ }^{3} J_{5,6}=11.93 \mathrm{~Hz}, 1 \mathrm{H}, 6-\mathrm{H}\right)$, $6.658\left(\mathrm{~d},{ }^{3} J_{8,9}=15.61 \mathrm{~Hz}, 1 \mathrm{H}, 8-\mathrm{H}\right), 6.739\left(\mathrm{dd},{ }^{3} J_{3,4}=11.47 \mathrm{~Hz}\right.$, $\left.{ }^{3} J_{4.5}=14.38 \mathrm{~Hz}, 1 \mathrm{H}, 4-\mathrm{H}\right), 6.995(\mathrm{~d}, 1 \mathrm{H}, 3-\mathrm{H}), 7.058(\mathrm{dd}, 1 \mathrm{H}$, $5-\mathrm{H}), 7.128(\mathrm{~d}, 1 \mathrm{H}, 9-\mathrm{H}), 7.704-7.836(\mathrm{~m}, 12 \mathrm{H}, m-, \mathrm{p}-\mathrm{Ph})$, $8.124-8.250(\mathrm{~m}, 8 \mathrm{H}, o-\mathrm{Ph}), 8.665\left(\mathrm{~d},{ }^{3} J_{17^{\prime}, 18^{\circ}}=4.84 \mathrm{~Hz}, 1 \mathrm{H}\right.$, $\left.18^{\prime}-\mathrm{H}\right), 8.746-8.830\left(\mathrm{~m}, 5 \mathrm{H}, 7^{\prime}-, 8^{\prime}-, 12^{\prime}-, 13^{\prime}-, 7^{\prime}-\mathrm{H}\right), 8.925(\mathrm{~d}, 1 \mathrm{H}$, $\left.3^{\prime}-\mathrm{H}\right), 9.488(\mathrm{~s}, 1 \mathrm{H}, 1-\mathrm{H}),-2.564(\mathrm{~s}, 2 \mathrm{H},=\mathrm{NH})$

\section{2,7,10,15-Tetramethyl-17-[2'-(5',10',15', 20'-tetraphenylpor-} phinyl)heptadecaoctaenal (14):

Wie für $11 \mathbf{i}$ beschrieben aus $6(0.57 \mathrm{mmol})$ in $\mathrm{MeOH}(4 \mathrm{~mL})$, Orthoameisensäure-methylester $(1.14 \mathrm{mmol}), \mathrm{TsOH}(0.5 \mathrm{mg})$ in $\mathrm{MeOH}(0.5 \mathrm{~mL}), 11 \mathrm{i}(0.19 \mathrm{mmol})$ in $\mathrm{CH}_{2} \mathrm{Cl}_{2}(30 \mathrm{~mL}), 1 \mathrm{~N} \mathrm{NaO}-$ Me-Lösung in $\mathrm{MeOH}(0.6 \mathrm{~mL})$. Ausbeute: $49 \% 14$ nach Umkristallisation aus $\mathrm{CH}_{2} \mathrm{Cl}_{2} / \mathrm{Hexan}$ als violettblaue Plättchen, $\mathrm{mp}$ $>270^{\circ} \mathrm{C}$.

$\mathrm{C}_{65} \mathrm{H}_{54} \mathrm{~N}_{4} \mathrm{O}$ ber. 906.4298 gef. 906.4302

${ }^{1} \mathrm{H}-\mathrm{NMR}$ (300 MHz, $\mathrm{CDCl}_{3} / \mathrm{TMS}$ ): all-E-Isomer $\delta=-2.549$ (s, $2 \mathrm{H},=\mathrm{NH}), 1.689\left(\mathrm{~s}, 3 \mathrm{H}, 15-\mathrm{CH}_{3}\right), 1.897\left(\mathrm{~s}, 3 \mathrm{H}, 2-\mathrm{CH}_{3}\right), 1.999(\mathrm{~s}$, $\left.3 \mathrm{H}, 10-\mathrm{CH}_{3}\right), 2.053\left(\mathrm{~s}, 3 \mathrm{H}, 7-\mathrm{CH}_{3}\right), 6.350-6.737(\mathrm{~m}, 9 \mathrm{H}, 4-, 6-, 8-$, 9- $\mathrm{H}, 11$ - bis $14-\mathrm{H}, 16-\mathrm{H}), 6.975\left(\mathrm{~d},{ }^{3} J_{3,4}=11.35 \mathrm{~Hz}, 1 \mathrm{H}, 3-\mathrm{H}\right)$ $7.057\left(\mathrm{dd},{ }^{3} J_{5,6}=11.95 \mathrm{~Hz},{ }^{3} J_{4,5}=14.75 \mathrm{~Hz}, 1 \mathrm{H}, 5-\mathrm{H}\right), 7.130$ $\left(\mathrm{d},{ }^{3} J_{16,17}=15.49 \mathrm{~Hz}, 1 \mathrm{H}, 17-\mathrm{H}\right), 7.721-7.813(\mathrm{~m}, 12 \mathrm{H}, m-, p-\mathrm{Ph})$, $8.114-8.241(\mathrm{~m}, 8 \mathrm{H}, \mathrm{OPh}), 8.648\left(\mathrm{~d},{ }^{3} J_{17^{\prime} .18^{\prime}}=4.80,1 \mathrm{H}, 18^{\prime}-\mathrm{H}\right)$, $8.727-8.813\left(\mathrm{~m}, 5 \mathrm{H}, 7^{\prime}-, 8^{\prime}-, 12^{\prime}-, 13^{\prime}-, 17^{\prime}-\mathrm{H}\right), 8.904$ (s, $\left.1 \mathrm{H}, 3^{\prime}-\mathrm{H}\right)$, $9.465(\mathrm{~s}, 1 \mathrm{H}, 1-\mathrm{H})$.

Wir danken der Deutschen Forschungsgemeinschaft und dem Fonds der Chemischen Industrie für die Unterstützung dieser Arbeit. Ferner danken wir der BASF AG für die großzügige Überlassung der Ausgangsverbindungen. Den Forschungspraktikanten J. Wonner und $H$. Strobel danken wir für ihre engagierte Mitarbeit.

Received: 15 January 1990

(1) Effenberger, F.; Schlosser, H.; Bäuerle, P.; Maier, S.; Port, H.; Wolf, H.C. Angew. Chem. 1988, 100, 274; Angew. Chem., Int. Ed. Engl. 1988, 27, 281.

(2) Schlosser, H. Diplomarbeit, Univ. Stuttgart, 1986. Schlosser, H. Teil der Dissertation Univ. Stuttgart, 1989.

(3) Arrhenius, T. S.; Blanchard-Desce, M.; Dvolaitzky, M.; Lehn, J. M.; Malthete, J. Proc. Natl. Acad. Sci. USA 1986, 83, 5355. Lehn, J.-M. Angew. Chem. 1988, 100 91; Angew. Chem., Int. Ed. Engl. 1988, 27, 89.

Blanchard-Desce, M.; Ledoux, I.; Lehn, J.-M.: Malthete, J.; Zyss, J. J. Chem. Soc., Chem. Commun. 1988, 737.

(4) Goehring, W.; Roth, S.; Hanack, M. Synth. Met. 1987, 17, 259.

(5) Carotenoids, Isler, O. (ed.), Birkhäuser Verlag, Basel, 1971. Carotenoid Chemistry and Biochemistry, Britton, G.; Goodwin, T.W. (Eds.), Pergamon Press, London, 1982.
(6) Reppe, K., in: Houben-Weyl, Vol. V/1 d, Georg Thieme Verlag, Stuttgart, 1972, p. 88.

Gosney, I.; Rowley, A.G. in: Organophosphorus Reagents in Organic Synthesis, Cadogan, I. G. (ed.), Academic Press, Inc., New York, 1979, p. 17.

(7) Reppe, K., in: Houben-Weyl, Vol. V/1 d, Georg-Thieme Verlag, Stuttgart, 1972, p. 18.

(8) Davies, A.J.; Khare, A.; Mallams, A.K.; Massy-Westropp, A.; Moss, G. P.; Weedon, B.C. L. J. Chem. Soc. Perkin Trans. 1 1984, 2147

Arm, C.; Pfander, H. Helv. Chim. Acta 1984, 67, 1540.

Aareskjold, K.; Liaaen-Jensen, S. Acta Chem. Scand. Ser. B 1984, 38, 43.

Bernhard, K.; Englert, G.; Mayer, H.; Müller, R.K.; Rüttimann, A.; Vecchi, M.; Widmer, E.; Zell, R. Helv. Chim. Acta 1981, 64, 2469.

Haag, A.; Eugster, C.H. Helv. Chim. Acta 1985, 68, 1897.

(9) Francis, G.W. Acta Chem. Scand. 1972, 26, 2969.

(10) Schlosser, M. Top. Stereochem. 1970, 5, 1.

Schlosser, M.; Schaub, B.; de Oliveira-Neto, J.; Jeganathan, S. Chimia 1986, 40, 244.

(11) Pauling, L. Fortschr. Chem. Org. Naturst. 1939, 3, 203.

(12) Weedon, B.C.L., in Carotenoids, Isler, O. (ed.), Birkhäuser Verlag, 1971, p. 267.

(13) Rüedi, P. Pure Appl. Chem. 1985, 57, 793.

(14) Kamber, M.; Pfander, H. J. Chromatogr. 1984, 295, 295.

(15) Zumbrunn, A.; Uebelhart, P.; Eugster, C. H. Helv. Chim. Acta $1985,68,1540$.

(16) Fabian, J.; Hartmann, H.: Light Absorption of Organic Colorants, Springer Verlag, Berlin, 1980, p. 24.

Das, P.K.; Becker, R.S. J. Phys. Chem. 1982, 86, 921.

Jaffé, H.H.; Orchin, M.: Theorie and Applications of UVSpectroscopy, John Wiley \& Sons, New York, 1962, p. 220. Hudson, B.S.; Kohler, B.E.; Schulten, K., in: Excited States, Vol. 6; Lim, E.C. (ed.), Academic Press, New York, 1982.

(17) Englert, G.; Vecchi, M. Helv. Chim. Acta 1980, 63, 1711.

(18) Vecchi, M.; Englert, G.; Maurer, R.; Meduna, V. Helv. Chim. Acta 1981, 64, 2746.

(19) Vetter, W.; Englert, G.; Rigassi, N.; Schwieter, U., in: Carotenoids, Isler, O. (ed.), Birkhäuser Verlag, 1971, p. 189.

(20) Englert, G., in: Carotenoid Chemistry and Biochemistry, Britton, G.; Goodwin, T.W. (eds.), Pergamon Press, London, 1982, p. 107.

(21) Die vollständigen ${ }^{1} \mathrm{H}-\mathrm{NMR}$-Daten für die Verbindungen $3 b-g, 4 b-f, 7 b-e, 11 e-h, 12 e$ und $15 g, h, 16 g$, h sind vom Autor oder von der Redaktion verfügbar.

(22) Barber, M.S.; Davis, J.B.; Jackman, L.M.; Weedon, B.C. L. J. Chem. Soc. 1960, 2870.

Schwieter, U.; Gutmann. H.; Lindlar, H.; Marbet, R.; Rigassi, N.; Rüegg, R.; Schaeren, S. F.; Isler, O. Helv. Chim. Acta 1966, 49,369 .

Moss, G.P. Pure Appl. Chem. 1976, 47, 97.

(23) Pattenden, G.; Way, J. E.; Weedon, B.C.L. J. Chem. Soc. C $1970,235$.

Bernhard, K.; Kienzle, F.; Mayer, H.; Müller, R. K. Helv. Chim. Acta 1980, 63, 1473.

(24) Inhoffen, H.H.; von der Bey, G. Liebigs Ann. Chem. 1953, $583,100$.

(25) Crossley, M. J.; Harding, M. M.; Sternhell, S. J. Am. Chem. Soc. 1986, $108,3608$.

Ponomarev, G. V.; Maravin, G. B. Chem. Heterocycl. Compd 1982, 18,50 .

(26) Callot, H.J. Tetrahedron 1973, 29, 899.

Callot, H.J.; Castro, B.; Selve, C. Tetrahedron Lett. 1978, 32 2877. 